\title{
VARIATIONS OF HAUSDORFF DIMENSION IN THE EXPONENTIAL FAMILY
}

\author{
Guillaume Havard, Mariusz Urbański and Michel Zinsmeister \\ Université Blaise Pascal, Laboratoire de Mathématiques \\ 63177 Aubière cedex, France; guillaume.havard@math.univ-bpclermont.fr \\ University of North Texas, Department of Mathematics \\ P.O. Box 311430, Denton TX 76203-1430, U.S.A.; urbanski@unt.edu \\ Université d'Orléans, Laboratoire de Mathématiques et Applications \\ B.P. 6759, 45067 Orléans cedex 2, France; Michel.Zinsmeister@math.cnrs.fr
}

\begin{abstract}
In this paper we deal with the following family of exponential maps $\left(f_{\lambda}: z \mapsto\right.$ $\left.\lambda\left(e^{z}-1\right)\right)_{\lambda \in[1,+\infty)}$. Denote by $d(\lambda)$ the hyperbolic dimension of $f_{\lambda}$. It is proved in [Ur, Zd $\left.\mathrm{d}^{1}\right]$ that the function $\lambda \mapsto d(\lambda)$ is real-analytic in $(1,+\infty)$, and in $\left[\mathrm{Ur}, \mathrm{Zd}^{2}\right]$ that it is continuous in $[1,+\infty)$. In this paper we prove that this map is $C^{1}$ on $[1,+\infty)$, with $d^{\prime}\left(1^{+}\right)=0$. Moreover we prove that depending on the value of $d(1)$

$$
\begin{cases}d^{\prime}(1+\varepsilon) \sim-\varepsilon^{2 d(1)-2} & \text { if } d(1)<\frac{3}{2} \\ \left|d^{\prime}(1+\varepsilon)\right| \lesssim-\varepsilon \log \varepsilon & \text { if } d(1)=\frac{3}{2} \\ \left|d^{\prime}(1+\varepsilon)\right| \lesssim \varepsilon & \text { if } d(1)>\frac{3}{2}\end{cases}
$$

In particular, if $d(1)<\frac{3}{2}$, then there exists $\lambda_{0}>1$ such that $d(\lambda)<d(1)$ for any $\lambda \in\left(1, \lambda_{0}\right)$.
\end{abstract}

\section{Introduction}

1.1. An overview of the problem. In this paper we deal with maps of the form $f_{\lambda}: z \mapsto \lambda\left(e^{z}-1\right)$ for $\lambda \geq 1$. As long as $\lambda$ is strictly greater than 1,0 is a repelling fixed point and there exists an attracting fixed point $q_{\lambda}<0$. Those two points collapse to 0 for $\lambda=1$, and 0 becomes parabolic. We are interested in $J_{\lambda}$, the set of points that do not escape to $\infty$ under iterations of $f_{\lambda}$. The Hausdorff dimension of this set, that we denote $d(\lambda)$, is an element of $(1,2)$, and is called the hyperbolic dimension of the map $f_{\lambda}$. While for any $\lambda$ the Julia set of $f_{\lambda}$ has Hausdorff dimension constant equal to 2 , cf. $\left[\mathrm{McMu}^{1}\right]$, the hyperbolic dimension varies with $\lambda$. Moreover, any invariant probability measure gives full mass to $J_{\lambda}$, and $d(\lambda)$ is, in the hyperbolic case, equal to the first zero of the pressure of the map $t \mapsto-t \log \left|f_{\lambda}^{\prime}\right|{ }^{1}$ cf. $\left[\mathrm{Ma}, \mathrm{Ur}^{1}\right]$.

Variations of $\lambda \mapsto d(\lambda)$ with respect to $\lambda$, is an interesting feature that reflects changes in geometry after perturbation of a dynamical system. The philosophy is that $d$ behaves smoothly, and even real-analytically, if we perturb a conformal hyperbolic dynamical system, in a real-analytic way.

This philosophy was proposed in 1981 Rio de Janeiro's conference by Sullivan [Su]. The same year Ruelle $[\mathrm{Ru}]$ proved that it was true for a class of hyperbolic

doi:10.5186/aasfm.2010.3523

2000 Mathematics Subject Classification: Primary 37F35, 37F45, 30D05.

Key words: Hausdorff dimension, Julia set, exponential family, parabolic points, thermodynamic formalism, conformal measures.

The research of Mariusz Urbański is partially supported by the NSF grant DMS 0700831.

${ }^{1}$ This result is known as Bowen's formula. 
conformal repellers. His strategy, used since then in other contexts, see $\left[\mathrm{Ur}_{\mathrm{Z}} \mathrm{Zd}^{1}\right]$ for the exponential family and $\left[\mathrm{Ma}, \mathrm{Ur}^{1}\right]$ and $\left[\mathrm{Ma}, \mathrm{Ur}^{2}\right]$ for meromorphic functions, was the following: prove a Bowen's formula that identifies the dimension as the zero of a pressure function, prove that this pressure is the logarithm of a simple and isolated eigenvalue of a Perron-Frobenius(-Ruelle) operator, then use some results about perturbation theory of operators.

When approaching the boundary of an hyperbolic components one can not expect any smoothness. Nevertheless there still exist some paths along which we still have continuity of the Hausdorff dimension. This was first proved by Bodart and Zinsmeister in [Bo,Zi] for the quadratic family, $z \mapsto z^{2}+c$, for $c \in \mathbf{R}$ approaching $\frac{1}{4}$ from the left. Then it has been proved for other parameters $c,[\mathrm{Ri}]$, or other rational maps, $\left[\mathrm{McMu}^{4}\right],[\mathrm{Bu}, \mathrm{Le}]$, or in other situations see $\left[\mathrm{McMu}^{3}\right]$ for Kleinian groups, $\left[\mathrm{Ur}, \mathrm{Zd}^{2}\right]$ for the exponential family. The strategy for such results is to control conformal measures, or Patterson-Sullivan measures, in order to prove that they converge towards the "good" conformal/Patterson-Sullivan measure. This usually boils down in proving that any limiting measure is non-atomic. Note that this strategy may also be used to prove discontinuity of the Hausdorff dimension, or more precisely to prove convergence towards something bigger than the Hausdorff dimension of the "limit set", [Do,Se,Zi], [Ur,Zi $\left.{ }^{1}\right]$ and $\left[\mathrm{Ur}, \mathrm{Zi}^{2}\right]$.

The problem of the derivative of the Hausdorff dimension has been, to our knowledge, investigated only in two other papers than the present one. In $\left[\mathrm{Ha}, \mathrm{Zi}^{1}\right]$ for the quadratic family it is proved that $d^{\prime}(c)$, the derivative of $d(c):=\operatorname{Hdim}\left(J_{c}\right)$, diverges towards $+\infty$ as $c$ converges towards $\frac{1}{4}$ from the left. In $\left[\mathrm{Ja}^{1}\right]$, still for the quadratic family, but this time for $c$ converging from the right towards $-\frac{3}{4}$, and under the realistic hypothesis that $d\left(-\frac{3}{4}\right)<\frac{4}{3}$, it is proved that $d^{\prime}(c)$ converges towards $-\infty$. In order to control the derivative the starting point in all those papers is first to get an exact formula for the derivative. This is done using thermodynamic formalism by differentiating the Bowen's formula. Then some uniform estimates of distortion in a neighborhood of the fixed point are used in order to control measures of fundamental annuli. Conclusions then come from a precise analysis of a certain integral. This is that last point that explains why such a study has not been done yet in a more general setting. In the present paper, as well as in $\left[\mathrm{Ha}, \mathrm{Zi}^{1}\right]$ and $\left[\mathrm{Ja}^{1}\right]$, some very particular properties of the case studied are used to conclude the theorem.

1.2. Main result. When one notes that if $\tau_{\lambda}$ denotes the translation by $-\lambda$, then we have $f_{\lambda} \circ \tau_{\lambda}=\tau_{\lambda} \circ g_{\lambda}$, with $g_{\lambda}(z)=\alpha(\lambda) e^{z}$ and $\alpha(\lambda)=\lambda e^{-\lambda}$, this philosophy (real analyticity of $d$ ) is in $\left[\mathrm{Ur}, \mathrm{Zd}^{1}\right]$ proved to be the case. More precisely, it is proved there that $d: \lambda \mapsto d(\lambda)$ is real-analytic on $(1,+\infty)$, and in $\left[\mathrm{Ur}^{2} \mathrm{Zd}^{2}\right]$, that it is continuous on $[1,+\infty)$. In this paper we study the asymptotic behavior of the function $\lambda \mapsto d^{\prime}(\lambda)$, and we prove the following.

Theorem 1.1. There exist $\lambda_{0}>1$ and $K>1$ such that for all $\lambda \in\left(1, \lambda_{0}\right)$

$$
\begin{cases}\frac{-1}{K}(\lambda-1)^{2 d(1)-2} \leq d^{\prime}(\lambda) \leq-K(\lambda-1)^{2 d(1)-2} & \text { if } d(1)<\frac{3}{2} \\ \left|d^{\prime}(\lambda)\right| \leq K(\lambda-1) \log \frac{1}{\lambda-1} & \text { if } d(1)=\frac{3}{2} \\ \left|d^{\prime}(\lambda)\right| \leq K(\lambda-1) & \text { if } d(1)>\frac{3}{2}\end{cases}
$$

In particular, the function $\lambda \mapsto d(\lambda)$ is $C^{1}$ on $[1,+\infty)$ with $d^{\prime}(1)=0$. 
Remark. As already mentioned, conjugating $f_{\lambda}$ by the translation $\tau_{\lambda}$, we get the family $g_{\lambda}:=\tau_{\lambda} \circ f_{\lambda} \circ \tau_{\lambda}^{-1}$, with $g_{\lambda}(z)=\lambda e^{-\lambda} e^{z}$. Changing variable to $\varepsilon:=\lambda e^{\lambda-1}-1$, we get the family $g_{\varepsilon}: z \mapsto(1+\varepsilon) e^{-1} e^{z}$ with $\varepsilon \sim(\lambda-1)$. Let $D(\varepsilon)$ be the hyperbolic dimension of $g_{\varepsilon}$, then

$$
\left\{\begin{aligned}
\left|D^{\prime}(\varepsilon)\right| \sim \varepsilon^{2 D(0)-3} & & \text { if } D(0)<\frac{3}{2}, \\
\left|D^{\prime}(\varepsilon)\right| \lesssim \log \frac{1}{\varepsilon} & & \text { if } D(0)=\frac{3}{2} \\
\left|D^{\prime}(\varepsilon)\right| \lesssim K & & \text { if } D(0)>\frac{3}{2} .
\end{aligned}\right.
$$

Note in particular that, in case $D(0)<\frac{3}{2}$, we get exactly the same asymptotic as the one in $\left[\mathrm{Ha}, \mathrm{Zi}^{1}\right]$ for the family $c \mapsto z^{2}+c$ with $c<\frac{1}{4}$. For this last family we were able to prove that $d\left(\frac{1}{4}\right)<\frac{3}{2}$, see [Ha,Se,Zi]. We do not know whether this inequality holds for the exponential family.

Note also that if $d(1)<\frac{3}{2}$, then we have a control over the sign of the derivative in a right hand sided neighborhood of 1 . It asserts that $d\left(1^{+}\right)$is a local maximum of the hyperbolic dimension.

Remark. There is to our knowledge no algorithm to compute accurately Hausdorff dimension of parabolic Julia sets. In [Ha,Se,Zi] an estimate of the Hausdorff dimension of the cauliflower (the Julia set of $z \mapsto z^{2}+\frac{1}{4}$ ) is done by calculating, with help of a computer, the first terms of an appropriate sum, then by estimating its tail. This method uses strongly particular properties of the map. More generally, one could build an infinite iterated function system whose limit set would have Hausdorff dimension equal to the hyperbolic dimension of the Julia set. Then, using results from [He,Ur], one could approximate this Hausdorff dimension by finite subsystems keeping track of the error. Finally, there are algorithms to calculate Hausdorff dimension of finite IFSs with any desired accuracy $\left[\mathrm{McMu}^{2}\right]$, [Je,Po]. However, to realize such program would be a tedious extremely time consuming task.

The proof of our main result will follow exactly along the same lines as the one of [Ha,Se,Zi], but will make an extensive use of the thermodynamic formalism for meromorphic functions, as developed by Urbański, Urbański and Kotus, Urbański and Zdunik, and Urbański and Mayer. The reader will find in $\left[\mathrm{Ma}, \mathrm{Ur}^{2}\right]$ all proofs of results we need in this paper, as well as a complete bibliography on the subject.

1.3. Organization of the paper. In the first part we use Chapter 8 of $\left[\mathrm{Ma}, \mathrm{Ur}^{2}\right]$ to get a formula for $d^{\prime}(\lambda)$, for any $\lambda \in(1,+\infty)$. This mainly consists of conjugating the dynamics and differentiating the pressure.

In the second part we collect some estimates of the distortion around the fixed point 0 . They are crucial since the formula obtained in the first part of this paper involves two integrals with respect to an invariant measure that has unbounded Radon-Nikodym derivative with respect to the Hausdorff measure in any neighborhood of 0 .

In the third part we use those estimates to control the integrals and to prove the main result.

In the first appendix we prove the estimates used in the second part of this paper in a more general setting than needed in this paper. Namely, we allow the repelling fixed point to converge towards a parabolic fixed point with several petals. The second appendix is devoted to the study of partial sums of some sequences that will be needed several times in the paper. 
Acknowledgements. The authors wish to thank the European Marie Curie network CODY which helped them to meet several times. They also thank the referee for his or her suggestions and careful reading of the paper.

\section{A formula for the derivative of the function $\lambda \mapsto d(\lambda)$}

Before giving and proving the formula of the derivative, which is done below in Proposition 2.1, we introduce some notation and recall some results concerning the thermodynamical formalism for that family of exponential maps.

2.1. Thermodynamic formalism. As it is done in $\left[\mathrm{Ur}_{\mathrm{Z}} \mathrm{Zd}^{1}\right]$ we associate to $f_{\lambda}$ the map $F_{\lambda}: P \rightarrow P$ defined by

$$
F_{\lambda} \circ \pi=\pi \circ f_{\lambda}
$$

with $\pi$ being the natural projection on the cylinder $P=\mathbf{C} / \sim$, with $z_{1} \sim z_{2}$ if and only if $\left(z_{1}-z_{2}\right)=2 i k \pi$, for some $k \in \mathbf{Z}$. Abusing slightly notation we will frequently identify $P$ with $\{z \in \mathbf{C}:-\pi<\operatorname{Im}(z) \leq \pi\}$. In particular, for any $z \in P$ we have $f_{\lambda}(z)=F_{\lambda}(z)$, and $F_{\lambda}(z)=F_{\lambda}\left(z^{\prime}\right)$ if and only if there exists $k \in \mathbf{Z}$ such that $f_{\lambda}(z)-f_{\lambda}\left(z^{\prime}\right)=2 i k \pi$. This tells us that for any $z \in P$, we have $F_{\lambda}^{-1}(z)=\left\{z_{k} \in\right.$ $\left.P \mid f_{\lambda}\left(z_{k}\right)=z+2 i k \pi, k \in \mathbf{Z}\right\}$. We also see that $J\left(F_{\lambda}\right)=\pi\left(J\left(f_{\lambda}\right)\right)=J\left(f_{\lambda}\right) \cap P$.

Let us now introduce some notation and collect some results, where we mainly refer to $\left[\mathrm{Ma}, \mathrm{Ur}^{2}\right]$, see also $\left[\mathrm{Ur}, \mathrm{Zd}^{1}\right],\left[\mathrm{Ur}, \mathrm{Zd}^{2}\right],[\mathrm{Ur}]$.

- For any $\lambda \geq 1$ we define $\mathscr{L}_{\lambda, t}$, the Perron-Frobenius operator associated with the potential $-t \log \left|F_{\lambda}^{\prime}\right|$. It acts on $\mathscr{H}_{\alpha}^{\lambda}$, the set of bounded $\alpha$-Hölder functions defined on $J\left(F_{\lambda}\right)$, in the following way, let $g \in \mathscr{H}_{\alpha}^{\lambda}$, and $z \in J\left(F_{\lambda}\right)$

$$
\begin{aligned}
\mathscr{L}_{\lambda, t}(g)(z) & =\sum_{F_{\lambda}(y)=z} \frac{1}{\left|\left(F_{\lambda}\right)^{\prime}(y)\right|^{t}} g(y) \\
& =\sum_{k \in \mathbf{Z}} \frac{1}{|z+\lambda+2 i k \pi|^{t}} g\left(z_{k}\right), \text { with } z_{k} \in P \text { such that } f_{\lambda}\left(z_{k}\right)=z+2 i k \pi .
\end{aligned}
$$

- The only $d(\lambda)$-conformal measure supported on $J_{\lambda}$ is denoted $m_{\lambda}{ }^{2}$

- The only equilibrium measure for the potential $-d(\lambda) \log \left|F_{\lambda}^{\prime}\right|$ and the dynamical system $\left(J_{\lambda}, F_{\lambda}\right)$ is denoted $\mu_{\lambda}$.

- The pressure of the potential $-t \log \left|F_{\lambda}^{\prime}\right|$ is denoted $P(\lambda, t)$, and is defined by

$$
P(\lambda, t)=\sup \left\{h_{\mu}-t \chi_{\mu}\right\}
$$

where the supremum is taken over all invariant probability measures $\mu$ supported on $J\left(F_{\lambda}\right)$ such that $\chi_{\mu}<+\infty$, where $h_{\mu}$ denotes the metric entropy of the measure $\mu$, and $\chi_{\mu}=\int \log \left|F_{\lambda}^{\prime}\right| d \mu$ is its Lyapunov exponent.

We will derive our formula for $d^{\prime}(\lambda)$ starting from Bowen's formula that asserts that for any $\lambda>1, d(\lambda)$ is the only real number so that $P(\lambda, d(\lambda))=0$ (see [Ur,Zd $\left.{ }^{1}\right]$ ). We want to differentiate this formula with respect to $\lambda$, and in order to do so we need to appropriately conjugate the dynamics of $F_{\lambda}$.

- Let $\lambda_{0}>1$ be fixed. For any $\lambda>1$, we denote by $h_{\lambda}$ the conjugating map from $J_{\lambda_{0}}$ to $J_{\lambda}$ such that $F_{\lambda} \circ h_{\lambda}=h_{\lambda} \circ F_{\lambda_{0}}$.

- We then set $\varphi_{\lambda, t}:=-t \log \left|F_{\lambda}^{\prime} \circ h_{\lambda}\right|$. It is a potential which is defined on $J_{\lambda_{0}}$. We then use Corollary 8.10 in $\left[\mathrm{Ma}, \mathrm{Ur}^{2}\right]$ that tells us that $(\lambda, t) \mapsto P_{0}\left(\varphi_{\lambda, t}\right)$ is real analytic

\footnotetext{
${ }^{2}$ We refer to section 3 of this paper for a definition and more details about conformal measures.
} 
for $\lambda$ close enough to $\lambda_{0}{ }^{3}$. Bowen's formula then implies that $\frac{\partial}{\partial \lambda} P_{0}\left(\varphi_{\lambda, d(\lambda)}\right)=0$. It is this calculation that leads to the desired formula.

2.2. The formula and its proof. In this section we prove the following formula

Proposition 2.1. For any $\lambda \in(1,+\infty)$ we have

$$
d^{\prime}(\lambda)=-\frac{d(\lambda)}{\chi_{\mu_{\lambda}}}\left(1-\frac{1}{\lambda}\right) \int_{J_{\lambda}} \sum_{k=1}^{+\infty} \operatorname{Re}\left(\frac{1}{\left(F_{\lambda}^{k}\right)^{\prime}}\right) d \mu_{\lambda},
$$

where $\mu_{\lambda}$ is the only equilibrium measure for the potential $-d(\lambda) \log \left|F_{\lambda}^{\prime}\right|$.

Let $\lambda_{0}>1$ be fixed and let $h_{\lambda}$ denote the conjugating map $F_{\lambda} \circ h_{\lambda}=h_{\lambda} \circ F_{\lambda_{0}}$. Since $\mu_{\lambda}$ is the equilibrium measure for the potential $-d(\lambda) \log \left|F_{\lambda}^{\prime}\right|$, we deduce that the potential $\varphi_{\lambda, d(\lambda)}$ has a unique equilibrium measure which is $\tilde{\mu}_{\lambda}:=h_{\lambda *}\left(\mu_{\lambda}\right)$. We shall now use Theorem 6.14 in $\left[\mathrm{Ma}, \mathrm{Ur}^{2}\right]$ which asserts that given a tame function $\varphi$ and a weakly tame function $\psi$ we have

$$
\frac{\partial}{\partial t} P_{0}(\varphi+t \psi)_{\mid t=0}=\int \psi d \mu_{\varphi}
$$

with $\mu_{\varphi}$ the equilibrium measure for the potential $\varphi$. We refer to chapter 4 of [Ma, $\mathrm{Ur}^{2}$ ] for definition of tame and loosely tame functions. By Lemma 8.9 in $\left[\mathrm{Ma}, \mathrm{Ur}^{2}\right]$, we know that for $R>0$ small enough, there exists $\beta>0$ such that $\forall \lambda \in\left(\lambda_{0}-R, \lambda_{0}+R\right)$ $\varphi_{\lambda, t}$ is $\beta$-tame. We then deduce from (2.2) that

$$
0=\frac{\partial}{\partial \lambda} P_{0}\left(\varphi_{\lambda, d(\lambda)}\right)=\int_{J_{\lambda_{0}}} \frac{\partial}{\partial \lambda}\left(\varphi_{\lambda, d(\lambda)}\right) d \tilde{\mu_{\lambda}} .
$$

We thus have to compute $\frac{\partial}{\partial \lambda} \varphi_{\lambda, d(\lambda)}$. Note that

$$
\varphi_{\lambda, d(\lambda)}=-d(\lambda) \log \left|F_{\lambda}^{\prime} \circ h_{\lambda}\right|=-d(\lambda)\left(\log \lambda+\operatorname{Re} h_{\lambda}\right) .
$$

Differentiating with respect to $\lambda$ we get

$$
\frac{\partial}{\partial \lambda} \varphi_{\lambda, d(\lambda)}=-d^{\prime}(\lambda) \log \left|F_{\lambda}^{\prime} \circ h_{\lambda}\right|-d(\lambda)\left(\frac{1}{\lambda}+\operatorname{Re} \frac{\partial}{\partial \lambda} h_{\lambda}\right) .
$$

Lemma 2.2. For any $\lambda \in(1,+\infty)$ and any $z \in J_{\lambda_{0}}$ we have

$$
\frac{\partial}{\partial \lambda} h_{\lambda}(z)=\left(1-\frac{1}{\lambda}\right) \sum_{k=1}^{+\infty} \frac{1}{\left(F_{\lambda}^{k}\right)^{\prime}\left(h_{\lambda}(z)\right)}-\frac{1}{\lambda} .
$$

In order to prove this formula we use two results from [Ur], Lemma 13.2 and Proposition 13.4, that we give in the following Lemma.

Lemma 2.3. For any $\lambda_{0} \in(1,+\infty)$ one can find $R>0, K>0$, and $\alpha>0$ such that

$$
\begin{gathered}
\forall \lambda \in B\left(\lambda_{0}, R\right) \quad \forall n \in \mathbf{N} \quad \forall z \in J_{\lambda} \quad\left|\left(F_{\lambda}^{n}\right)^{\prime}(z)\right| \geq K(1+\alpha)^{n}, \\
\forall \lambda \in B\left(\lambda_{0}, R\right) \quad \forall z \in J_{\lambda} \quad\left|\frac{\partial}{\partial \lambda} h_{\lambda}(z)\right|<K .
\end{gathered}
$$

\footnotetext{
${ }^{3}$ We denote here $P_{0}$ the pressure with respect to the dynamical system $\left(J_{\lambda_{0}}, F_{\lambda_{0}}\right)$.
} 
We can now prove Lemma 2.2.

Proof. In order to simplify notation, we write $\dot{h}_{\lambda}$ instead of $\frac{\partial}{\partial \lambda} h_{\lambda}$, and we drop $z$. We start with the conjugating formula $h_{\lambda} \circ F_{\lambda_{0}}=F_{\lambda} \circ h_{\lambda}=\lambda\left(e^{h_{\lambda}}-1\right)$, that we differentiate with respect to $\lambda$. We thus get

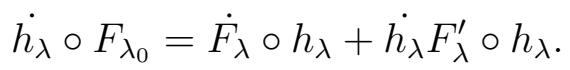

So that we have

$$
\dot{h_{\lambda}}=\frac{\dot{h_{\lambda}} \circ F_{\lambda_{0}}}{F_{\lambda}^{\prime} \circ h_{\lambda}}-\frac{\dot{F_{\lambda}} \circ h_{\lambda}}{F_{\lambda}^{\prime} \circ h_{\lambda}} .
$$

Iterating this formula we end up for $n \in \mathbf{N}$ with

$$
\dot{h_{\lambda}}(z)=\frac{\dot{h_{\lambda} \circ F_{\lambda_{0}}^{n}}}{\left(F_{\lambda}^{n}\right)^{\prime} \circ h_{\lambda}}-\sum_{k=1}^{n} \frac{\dot{F_{\lambda} \circ F_{\lambda}^{k-1} \circ h_{\lambda}}}{\left(F_{\lambda}^{k}\right)^{\prime} \circ h_{\lambda}} .
$$

Using Lemma 2.3 we deduce that

$$
\frac{\dot{h_{\lambda}}\left(F_{\lambda_{0}}^{n}(z)\right)}{\left(F_{\lambda}^{n}\right)^{\prime}\left(h_{\lambda}(z)\right)} \quad \text { is converging towards } 0 \text {. }
$$

On the other hand, since $\dot{F}_{\lambda}(z)=e^{z}-1=\frac{1}{\lambda} F_{\lambda}^{\prime}(z)-1$, for any $k$ we have

$$
\frac{\dot{F}_{\lambda} \circ F_{\lambda}^{k-1}}{\left(F_{\lambda}^{k}\right)^{\prime}}=\frac{1}{\lambda} \frac{1}{\left(F_{\lambda}^{k-1}\right)^{\prime}}-\frac{1}{\left(F_{\lambda}^{k}\right)^{\prime}} \text {. }
$$

This leads to

$$
\sum_{k=1}^{n} \frac{\dot{F}_{\lambda} \circ F_{\lambda}^{k-1}}{\left(F_{\lambda}^{k}\right)^{\prime}}=\frac{1}{\lambda}-\frac{1}{\left(F_{\lambda}^{n}\right)^{\prime}}+\left(\frac{1}{\lambda}-1\right) \sum_{k=1}^{n-1} \frac{1}{\left(F_{\lambda}^{k}\right)^{\prime}} .
$$

Using (2.6) in Lemma 2.3 we get that the series on the left above is converging towards

which finishes the proof.

$$
\sum_{k=1}^{+\infty} \frac{1}{\left(F_{\lambda}^{k}\right)^{\prime}}
$$

Using (2.4) and Lemma 2.2 in (2.3) we get

$$
-d^{\prime}(\lambda) \int_{J_{\lambda_{0}}} \log \left|F_{\lambda}^{\prime}\right| d \tilde{\mu}_{\lambda}-d(\lambda)\left(1-\frac{1}{\lambda}\right) \int_{J_{\lambda_{0}}} \operatorname{Re} \sum_{k \geq 1} \frac{1}{\left(F_{\lambda}^{k}\right)^{\prime} \circ h_{\lambda}}=0 .
$$

For any function $g$ continuous on $J_{\lambda}$ we have $\tilde{\mu}_{\lambda}\left(g \circ h_{\lambda}\right)=\mu_{\lambda}(g)$. We deduce from (2.8) that Proposition 2.1 is true.

\section{Local dynamic and uniform estimates}

In this section we introduce some notations and collect estimates proved in the appendix in a more general setting. ${ }^{4}$ We then use these estimates in order to control uniformly conformal measures $\left(m_{\lambda}\right)$ and equilibrium measures $\left(\mu_{\lambda}\right)$.

3.1. Notation. We know that $J_{\lambda} \cap P \subset\left\{z \in \mathbf{C} \mid-\frac{\pi}{2}<\operatorname{Im} z<\frac{\pi}{2}\right\}$. Given $0<\theta<\frac{\pi}{2}$ we denote by $S_{\theta}$ the sector $\left\{r e^{i \alpha} \mid r>0,-\theta<\alpha<\theta\right\}$. For $r_{0}<<1$

\footnotetext{
${ }^{4}$ We deal in the appendix with a family of germs of holomorphic functions in a neighborhood of a repelling fixed point which degenerates into a parabolic fixed point with $p$ petals.
} 
we fix $0<\theta<\frac{\pi}{2}$ to be such that $J_{1} \cap B(0, r) \subset S_{\theta}$. Then we choose $\varepsilon_{0}>0$ small enough so that for any $0 \leq \lambda=1+\varepsilon \leq \lambda_{0}=1+\varepsilon_{0}$ we have $f_{\lambda}^{-1}\left(S_{\theta}\right) \subset S_{\theta}$ and $J_{\lambda} \cap B(0, r) \subset S_{\theta}$. We then set $\gamma_{0}=\left\{r_{0} e^{i t} \mid t \in\right]-\theta, \theta[\}, \gamma_{1}(\lambda)=f_{\lambda}^{-1}\left(\gamma_{0}\right)$. Joining $r_{0} e^{i \theta}$ with $f_{\lambda}^{-1}\left(r_{0} e^{i \theta}\right)$ by a line, and doing the same with $r_{0} e^{-i \theta}$ and its image by $f_{\lambda}^{-1}$, we get a cell $C_{0}(\lambda)$. It is a simply connected domain. A compactness argument tells us that if $1 \leq \lambda \leq \lambda_{0}$, then there exists a simply connected domain $V \subset S_{\theta}$ such that the closure of $\cup_{\lambda} C_{0}(\lambda)$ is a subset of $V$. In particular, Koebe's Distortion Theorem gives us a constant $K>1$, only depending on $r_{0}$ and $\lambda_{0}$, such that for any univalent function $h$ on $V$ and any point $x$ and $y$ in $\overline{\cup_{\lambda} C_{0}(\lambda)}$ we have $\frac{1}{K} \leq \frac{\left|h^{\prime}(x)\right|}{\left|h^{\prime}(y)\right|} \leq K$. We will use later on this fact with inverse branches of $f_{\lambda}^{n}$. They are well defined on $V$ since the post-singular set of the $f_{\lambda}$ 's, i.e. the orbit $-\lambda$ under $f_{\lambda}$, is a subset of $(-\infty, 0)$.

We then define for each integer $n$ the set $C_{n}(\lambda):=f_{\lambda}^{-n}\left(C_{0}(\lambda)\right)$, with $f_{\lambda}^{-n}$ being the $n^{\text {th }}$ iterates of the inverse branch of $f_{\lambda}$ defined on $B\left(0, r_{0}\right)$ that fixes 0 . In the following we are working with respect to measures concentrated on $J_{\lambda}$ of dimension strictly greater to 1 . One checks easily in that context that with respect to such measure $\left(C_{n}(\lambda)\right)_{n \in \mathrm{N} \cup\{0\}}$ is a partition of $B\left(0, r_{0}\right)$. Moreover the set $C_{0}(\lambda)$ is mapped univalently by $f_{\lambda}^{-n}$ to $C_{n}(\lambda)$.

Let $N_{\varepsilon}$ be an integer ${ }^{5}$ and defined the sequence $\left(a_{n, \varepsilon}\right)_{n \in \mathbf{N}}$ as $a_{n, \varepsilon}=\frac{1}{n}$ if $n \leq N_{\varepsilon}$, and $a_{n, \varepsilon}=\varepsilon(1+\varepsilon)^{-n}$ if $n \geq N_{\varepsilon}$. Note that $a_{n, \varepsilon} \rightarrow 0$. In order to simplify notations, we let $a_{n}:=a_{n, \varepsilon}$. We consider now the one parameter familly of sequences, $\left(a_{n}(\alpha)\right)_{n \in \mathbf{N}}$, defined for $n \in \mathbf{N}$ by $a_{n}(\alpha):=a_{n}^{\alpha}$. We are also interested in partial sums of $\sum a_{n}(\alpha)$. For $k \leq n$ we let $S_{k, n}(\alpha):=\sum_{l=k}^{n} a_{l}(\alpha)$. The sequence $\left(a_{n}(\alpha)\right)$ will describe, for different values of $\alpha$, the distortion around 0 , the conformal measure of partition sets of a neighborhood of 0 , and the partial sums $S_{k, n}(\alpha)$ will play a role in controlling the invariant measure of the same partition sets, as well as evaluating the integral which is crucial in order to get our main result. Those estimates are easy and we use them in this section but we postponed their proofs to the appendix.

3.2. Uniform estimates of the distortion. In this section we give uniform estimates depending on $\lambda$ for the local dynamics next to the repelling-parabolic fixed point 0 . We recall that the family we are studying is given for $\lambda:=1+\varepsilon \geq 1$ by $f_{\lambda}(z)=\lambda\left(e^{z}-1\right)$. In particular, in a neighborhood of 0 , the local dynamic is given by the following Taylor expansion

$$
F_{\lambda}(z)=f_{\lambda}(z)=\lambda z+z^{2}+z^{3} g_{\lambda}(z)
$$

With $g_{\lambda}(z)$ uniformly bounded, independently of $\lambda$, as soon as a neighborhood of zero has been fixed. Note in particular that for $\varepsilon=0$, the point 0 is a parabolic fixed point with one petal.

We apply the general results of the first appendix of this paper to this special family $f_{\lambda}$. In the remaining of the paper we set $\lambda=1+\varepsilon$ and we denote the relevant quantities by indexing them equally well either by $\varepsilon$ or $\lambda$. Moreover, in the remainder of this section $F_{\lambda}^{-n}$ will be the inverse branch of $F_{\lambda}^{-n}$ that fixes 0 . From Proposition 5.7 we deduce that

\footnotetext{
${ }^{5}$ In our study we have $N_{\varepsilon} \sim \frac{1}{\varepsilon}=\frac{1}{\lambda-1}$.
} 
Proposition 3.1. Let $0<r_{0}, 1<\lambda_{0}$ be fixed. Then there exists $K>1$ such that $\forall \lambda \in\left(1, \lambda_{0}\right), \forall z \in C_{0}(\lambda)$, and $\forall n \in \mathbf{N}$

$$
\frac{1}{K} a_{n}(2) \leq\left|\left(F_{\lambda}^{-n}\right)^{\prime}(z)\right| \leq K a_{n}(2) .
$$

The following result is technical but will be crucial in order to control the sign of the derivative $d^{\prime}(\lambda)$.

Lemma 3.2. Let $0<r_{0}, 1<\lambda_{0}$ be fixed. There exists an integer $N$ such that $\forall n>N, \forall k \in \mathbf{N} \cap[1, n-N], \forall \lambda \in\left(1, \lambda_{0}\right)$ and $\forall z \in C_{n}(\lambda)$

$$
\frac{\sqrt{3}}{2}\left|\left(F_{\lambda}^{k}\right)^{\prime}(z)\right| \leq \operatorname{Re}\left(F_{\lambda}^{k}\right)^{\prime}(z) .
$$

Proof. Let $z \in C_{n}$ and $\theta_{k}(z)=\arg \left(F_{\lambda}^{k}\right)^{\prime}(z)$. The Lemma boils down to proving that $\left|\theta_{k}(z)\right| \leq \frac{\pi}{6}$. One computes that

$$
\left(F_{\lambda}^{k}\right)^{\prime}(z)=\prod_{j=0}^{k-1} F_{\lambda}^{\prime}\left(F_{\lambda}^{j}(z)\right)=\lambda^{k} \exp \left(\sum_{j=0}^{k-1} F_{\lambda}^{j}(z)\right) .
$$

So that we have $\theta_{k}(z)=\sum_{j=0}^{n-1} \operatorname{Im}\left(F_{\lambda}^{j}(z)\right)$. Since $F_{\lambda}^{j}(z)$ belongs to $C_{n-j}$, we may use Corollary 5.8 which asserts that $|\operatorname{Im}(Z)| \lesssim \frac{1}{(n-j)^{2}}$ for any $Z \in C_{n-j}$. We thus have

$$
\left|\theta_{k}(z)\right| \lesssim \sum_{j=1}^{n-k} \frac{1}{(n-j)^{2}} \leq \sum_{j=N}^{+\infty} \frac{1}{j^{2}}
$$

This is less than $\frac{\pi}{6}$ if $N$ is big enough and we are done.

We end this section with two more estimates of the distortion. The first one needs the following observation on the localization of $J\left(f_{\lambda}\right)$.

Lemma 3.3. For every $R>0$ there exists $\Delta>0$ such that for all $\lambda>1$,

$$
J\left(f_{\lambda}\right) \backslash \bigcup_{n=-\infty}^{+\infty} B(2 \pi n i, R) \subset\{z \in \mathbf{C}: \operatorname{Re} z \geq \Delta\} .
$$

Proof. First notice that

$f_{\lambda}(\{z \in \mathbf{C}: \operatorname{Re} z<0\})=B(-\lambda, \lambda) \subset\{z \in \mathbf{C}: \operatorname{Re} z<1-\lambda\} \subset\{z \in \mathbf{C}: \operatorname{Re} z<0\}$. Thus

$$
\{z \in \mathbf{C}: \operatorname{Re} z<0\} \subset \mathscr{F}\left(f_{\lambda}\right):=\text { Fatou set of } f_{\lambda} .
$$

Now write $z=x+i y$. Then

$$
\operatorname{Re}\left(f_{\lambda}(z)\right)=\operatorname{Re}\left(\lambda\left(e^{x} \cos y+i e^{x} \sin y-1\right)\right)=\lambda\left(e^{x} \cos y-1\right) .
$$

Note that there exists $\Delta_{1}>0$ so small that if $0<x<\Delta_{1}$ and $x+i y \notin$ $\bigcup_{n=-\infty}^{+\infty} B(2 \pi n i, R)$, then $\operatorname{dist}(y,\{2 \pi n i: n \in \mathbf{Z}\})>R / 2$, and consequently, $\cos y<$ $\cos (R / 2)$. Hence, $\operatorname{Re}\left(f_{\lambda}(z)\right)<\lambda\left(e^{\Delta_{1}} \cos (R / 2)-1\right)$. Take now $0<\Delta \leq \Delta_{1}$ so small that $e^{\Delta} \cos (R / 2)<1$. So $\operatorname{Re}\left(f_{\lambda}(z)\right)<0$ and, by $(3.1), f_{\lambda}(z) \in \mathscr{F}\left(f_{\lambda}\right)$. Therefore we have proved that

$$
\left\{z \in \mathbf{C} \backslash \bigcup_{n=-\infty}^{+\infty} B(2 \pi n i, R): \operatorname{Re} z<\Delta\right\} \subset \mathscr{F}\left(f_{\lambda}\right) .
$$


We are done.

Now notice that if $\operatorname{Re} z \geq \Delta$, then

$$
\left|f_{\lambda}^{\prime}(z)\right|=\lambda e^{\operatorname{Re} z} \geq \lambda e^{\Delta}>1 .
$$

Combining this and Lemma 3.3, we obtain the following.

Lemma 3.4. For every $R>0$ there exists $\gamma>1$ such that for every $z \in$ $J\left(F_{\lambda}\right) \backslash B(0, R)$,

$$
\left|F_{\lambda}^{\prime}(z)\right| \geq \gamma
$$

Using Proposition 5.7, Lemma 3.4 and the same reasoning as for the proof of Lemma 3.6 in $\left[\mathrm{Ha}, \mathrm{Zi}^{1}\right]$ we prove the following result.

Lemma 3.5. There exist $0<r_{0}, 1<\lambda_{0}$ and $1<K$ such that $\forall \lambda \in\left(1, \lambda_{0}\right)$ and $\forall z \in J_{\lambda}$,

$$
F_{\lambda}^{n}(z) \notin B\left(0, r_{0}\right) \Rightarrow K n^{2} \leq\left|\left(F_{\lambda}^{n}\right)^{\prime}(z)\right| .
$$

3.3. Conformal measures. Let us recall that a probability measure $m_{\lambda}$ is called conformal if its strong Jacobian is equal to $\left|F_{\lambda}^{\prime}\right|^{d(\lambda)}$. This means that for any measurable set $A$ on which $f_{\lambda}$ is $1-1$ we have

$$
m_{\lambda}\left(F_{\lambda}(A)\right)=\int_{A}\left|F_{\lambda}^{\prime}\right|^{d(\lambda)} d m_{\lambda} .
$$

Those measures are usually a powerful tool to study Hausdorff dimension of Julia sets. In fact their definition is dynamical but they very often carry a geometrically significant information about the Julia set. In many of cases they coincide (up to a multiplicative constant) with Hausdorff or packing measures on the Julia set.

Using Proposition 5.7 and the notation introduced below we get the following.

Proposition 3.6. Let $0<r_{0}, 1<\lambda_{0}$ be fixed. Then there exists $K>1$ such that $\forall \lambda \in\left(1, \lambda_{0}\right)$, and $\forall n \in \mathbf{N}$

$$
\frac{1}{K} a_{n}(2 d(\lambda)) \leq m_{\lambda}\left(C_{n}(\lambda)\right) \leq K a_{n}(2 d(\lambda)) .
$$

Proof. This is not difficult when one observes that for each $\lambda$ the function $F_{\lambda}^{n}$ is univalent on $C_{n}(\lambda)$. In particular using the definition of a conformal measure we deduce that

$$
m_{\lambda}\left(C_{0}(\lambda)\right)=m_{\lambda}\left(F_{\lambda}^{n}\left(C_{n}(\lambda)\right)\right)=\int_{C_{n}(\lambda)}\left|\left(F_{\lambda}^{n}\right)^{\prime}\right|^{d(\lambda)} d m_{\lambda}
$$

We then use estimates of Proposition 3.1, since $\left|\left(F_{\lambda}^{n}\right)^{\prime}\right|$ on $C_{n}(\lambda)$ is comparable with $\left|\left(F_{\lambda}^{-n}\right)^{\prime}\right|^{-1}$ on $C_{0}(\lambda)$. We deduce that there exists a constant $K>0$ such that for any $z \in C_{0}(\lambda)$

$$
\frac{1}{K}\left|\left(F_{\lambda}^{n}\right)^{\prime}(z)\right|^{-d(\lambda)} \leq m_{\lambda}\left(C_{n}(\lambda)\right) \leq K\left|\left(F_{\lambda}^{n}\right)^{\prime}(z)\right|^{-d(\lambda)} .
$$

We can now conclude the proof by using again Proposition 5.7.

Remark. Let $m_{\infty}$ be any accumulation point of the family of probability measures $\left(m_{\lambda}\right)_{\lambda>1}$. Let $\left(\lambda_{n}\right)$ be a sequence of real numbers converging from above towards 1 such that the sequence $\left(m_{\lambda_{n}}\right)$ converges weakly to $m_{\infty}$, and $\left(d\left(\lambda_{n}\right)\right)$ converges to 
some $d \geq 0$. For any $r>0$ small enough one may find $N(r)$ such that

$$
\forall n \geq N(r) \quad B(0, r) \cap J_{\lambda_{n}} \subset\{0\} \cup \bigcup_{k \leq N(r)} C_{k}\left(\lambda_{n}\right) .
$$

In particular, we conclude if $r>0$ is such that $m_{\infty}(\{|z|=r\})=0$, that

$$
m_{\infty}(B(0, r))=\lim _{n \rightarrow \infty} m_{\lambda_{n}}(B(0, r)) \leq \lim _{n \rightarrow \infty} \sum_{k>N(r)} m_{\lambda_{n}}\left(C_{k}\left(\lambda_{n}\right)\right) \leq \frac{K}{N(r)^{2 d\left(\lambda_{n}\right)-1}} .
$$

So that we conclude that $m_{\infty}$ has no atom at 0 . It is one of the main points in our argument that $d(\lambda) \rightarrow d(1)$ when $\lambda \rightarrow 1$, see [Ur, Zd $\left.{ }^{2}\right]$.

We end this section about conformal measures with a technical Lemma. It will be used in the next section concerning invariant measures.

Before stating and proving this result we recall that $P=\{z \in \mathbf{C} \mid-\pi<$ $\operatorname{Im} z \leq \pi\}$, and for any $M>0$, and any $r>0$, we introduce the following notation: $P_{M}:=\{z \in P \mid \operatorname{Re} z \leq M\}, \mathscr{B}_{r}:=P \backslash B(0, r)$ and $\mathscr{B}_{r, M}:=P_{M} \cap \mathscr{B}_{r}$.

Lemma 3.7. There exist $0<\alpha<\beta$ such that $\forall M \geq 2, \forall \lambda \in\left[1, \lambda_{0}\right]$, with $\left.\left.\lambda_{0}<\frac{\pi}{3}, \forall r \in\right] 0, \frac{\pi}{3}-\lambda_{0}\right]$ and $\forall A \subset B(0, r)$ measurable, we have

$$
\alpha m_{\lambda}(A) \leq m_{\lambda}\left(F_{\lambda}^{-1}(A) \cap \mathscr{B}_{r}\right) \leq \beta m_{\lambda}(A)
$$

and

$$
m_{\lambda}\left(F_{\lambda}^{-1}(A) \cap \mathscr{B}_{r, M}\right) \leq m_{\lambda}\left(F_{\lambda}^{-1}(A) \cap \mathscr{B}_{r}\right) \leq 54 \beta m_{\lambda}\left(F_{\lambda}^{-1}(A) \cap \mathscr{B}_{r, M}\right) .
$$

Proof. Let $B_{k}$ be the connected component of $F_{\lambda}^{-1}(B(0, r))$ such that $f_{\lambda}\left(B_{k}\right)=$ $B(2 i k \pi, r)$. For any $z \in B_{k}$ we have

$$
\left|F_{\lambda}^{\prime}(z)\right|=\left|f_{\lambda}^{\prime}(z)\right|=\left|f_{\lambda}(z)+\lambda\right|=\left|\lambda+2 i k \pi+a e^{i \theta}\right|,
$$

with $a<r$ and $0 \leq \theta<2 \pi$. With our assumptions this leads, for $|k| \geq 1$, to

$$
2|k| \pi-\frac{\pi}{3} \leq\left|F_{\lambda}^{\prime}(z)\right|=\left|f_{\lambda}^{\prime}(z)\right| \leq 2|k| \pi+\frac{\pi}{3} .
$$

Since $\left|f_{\lambda}^{\prime}(z)\right|=\lambda \exp (\operatorname{Re} z)$, we also get, for any $|k| \geq 1$, that

$$
\forall z \in B_{k} \quad \log 5 \leq \operatorname{Re} z .
$$

As a consequence we see that $F_{\lambda}^{-1}(B(0, r)) \cap \mathscr{B}_{r}=\bigcup_{|k| \geq 1} B_{k}$.

The measure $m_{\lambda}$ being conformal we have

$$
m_{\lambda}(A)=m_{\lambda}\left(F_{\lambda}\left(A_{k}\right)\right)=\int_{A_{k}}\left|F_{\lambda}^{\prime}\right|^{d(\lambda)} d m \lambda,
$$

with $A_{k}:=F_{\lambda}^{-1}(A) \cap B_{k}$. From (3.8) we deduce that

$$
\frac{m_{\lambda}(A)}{\left(2|k| \pi+\frac{\pi}{3}\right)^{d(\lambda)}} \leq m_{\lambda}\left(A_{k}\right) \leq \frac{m_{\lambda}(A)}{\left(2|k| \pi-\frac{\pi}{3}\right)^{d(\lambda)}},
$$

so that

$$
2 m_{\lambda}(A) \sum_{k \geq 1} \frac{1}{\left(2 k \pi+\frac{\pi}{3}\right)^{d(\lambda)}} \leq m_{\lambda}\left(F_{\lambda}^{-1}(A) \cap \mathscr{B}_{r}\right) \leq 2 m_{\lambda}(A) \sum_{k \geq 1} \frac{1}{\left(2 k \pi-\frac{\pi}{3}\right)^{d(\lambda)}}
$$


The function $\lambda \mapsto d(\lambda)$ being continuous on $\left[1, \lambda_{0}\right]$ one may consider its minimum $\delta$ which is strictly greater than 1 . With $\alpha=2 \sum_{k \geq 1} \frac{1}{\left(2 k \pi+\frac{\pi}{3}\right)^{2}}$ and $\beta=2 \sum_{k \geq 1} \frac{1}{\left(2 k \pi-\frac{\pi}{3}\right) \delta}$ we have

$$
\alpha m_{\lambda}(A) \leq m_{\lambda}\left(F_{\lambda}^{-1}(A) \cap \mathscr{B}_{r}\right) \leq \beta m_{\lambda}(A)
$$

This is (3.5).

Note that (3.8) tells us that for any $z \in B_{1}$ we have $\operatorname{Re} z \leq \log \left(2 \pi+\frac{\pi}{3}\right)<2 \leq M$. This implies that $B_{1} \subset \mathscr{B}_{r, M}$. In particular, we have

$$
m_{\lambda}\left(A_{1}\right) \leq m_{\lambda}\left(F_{\lambda}^{-1}(A) \cap \mathscr{B}_{r, M}\right) .
$$

We then deduce from (3.9) that

$$
\frac{m_{\lambda}(A)}{\left(2 \pi+\frac{\pi}{3}\right)^{2}} \leq m_{\lambda}\left(F_{\lambda}^{-1}(A) \cap \mathscr{B}_{r, M}\right) .
$$

Together with (3.5) we conclude that

$$
m_{\lambda}\left(F_{\lambda}^{-1}(A) \cap \mathscr{B}_{r}\right) \leq\left(2 \pi+\frac{\pi}{3}\right)^{2} \beta m_{\lambda}\left(F_{\lambda}^{-1}(A) \cap \mathscr{B}_{r, M}\right) .
$$

Since $\left(2 \pi+\frac{\pi}{3}\right)^{2} \leq 54$, we conclude that the left hand side inequality of (3.6) holds. The right hand side being obvious the proof is finished.

3.4. Invariant measures. Let us first recall that $\mu_{\lambda}=\rho_{\lambda} m_{\lambda}$ is the unique $F_{\lambda^{-}}$ invariant probability measure equivalent with $m_{\lambda}$. This measure is also the unique equilibrium state for the potential $-d(\lambda) \log \left|F_{\lambda}^{\prime}\right|$, i.e.,

$$
h_{\mu_{\lambda}}-d(\lambda) \int \log \left|F_{\lambda}^{\prime}\right| d \mu_{\lambda}=\sup \left\{h_{\mu}-d(\lambda) \int \log \left|F_{\lambda}^{\prime}\right| d \mu\right\},
$$

where supremum is taken over all $F_{\lambda}$-invariant ergodic probability measures such that $\int \log \left|F_{\lambda}^{\prime}\right| d \mu<+\infty$. The function $\rho_{\lambda}$ is obtained in $\left[\mathrm{Ma}, \mathrm{Ur}^{2}\right]$ as the limit of the sequence $\mathscr{L}_{\lambda}^{n}(1)$. The main results of this section is

Proposition 3.8. Let $0<r_{0}, 1<\lambda_{0}$ being fixed. Then there exists $K>1$ such that $\forall \lambda \in\left(1, \lambda_{0}\right)$, and $\forall n \in \mathbf{N}$

$$
\begin{aligned}
& \text { (i) } \frac{1}{K} a_{n}(2 d(\lambda)-1) \leq \mu_{\lambda}\left(C_{n}(\lambda)\right) \leq K a_{n}(2 d(\lambda)-1) \text { if } n \leq N_{\varepsilon} . \\
& \text { (ii) } \frac{1}{K} \frac{a_{n}(2 d(\lambda))}{\varepsilon} \leq \mu_{\lambda}\left(C_{n}(\lambda)\right) \leq K \frac{a_{n}(2 d(\lambda))}{\varepsilon} \text { if } n \geq N_{\varepsilon} .
\end{aligned}
$$

Proof. Let $\mathscr{B}_{r}:=P \backslash B(0, r)$. We know that $\mu_{\lambda}\left(\mathscr{B}_{r}\right)>0$ so that the first return time $N_{\lambda, r}(z):=\inf \left\{n \geq 1 \mid F_{\lambda}^{n}(z) \in \mathscr{B}_{r}\right\}$ is finite $\mu_{\lambda}$-almost-surely. Let $\mathscr{B}_{\lambda, n}:=\left\{N_{\lambda, r}=n\right\}$. We recall that the sets $\left(C_{n}\right)$ are introduced at the beginning of this section. Note that for $r$ small enough we have $\mathscr{B}_{\lambda, n} \cap B(0, r)=C_{n-1}(\lambda)$. Since $\mu_{\lambda}$ is $F_{\lambda}$-invariant its restriction to $\mathscr{B}_{r}$ is invariant for the first return map in $\mathscr{B}_{r}$, that we denote $T_{\lambda}$. Moreover, $\mu_{\lambda}$ can be built from this $T_{\lambda}$-invariant measure and this leads, for any measurable set $A$, to the formula

$$
\mu_{\lambda}(A)=\sum_{n \geq 1} \sum_{k=0}^{n-1} \mu_{\lambda}\left(F_{\lambda}^{-k}(A) \cap \mathscr{B}_{\lambda, n} \cap \mathscr{B}_{r}\right) .
$$


We are interested in the sets $C_{l}$ for which we get

$$
\mu_{\lambda}\left(C_{l}\right)=\sum_{n \geq 1} \sum_{k=0}^{n-1} \mu_{\lambda}\left(F_{\lambda}^{-k}\left(C_{l}\right) \cap \mathscr{B}_{\lambda, n} \cap \mathscr{B}_{r}\right) .
$$

Note now that the set $F_{\lambda}^{-k}\left(C_{l}\right) \cap \mathscr{B}_{\lambda, n} \cap \mathscr{B}_{r}$ is empty unless $n>l+1$ and $k=n-l-1$. In this case we have $F_{\lambda}^{-(n-l)}\left(C_{l}\right) \cap \mathscr{B}_{\lambda, n} \cap \mathscr{B}_{r}=F_{\lambda}^{-1}\left(C_{n-2}\right) \cap \mathscr{B}_{r}$. We thus conclude that

$$
\mu_{\lambda}\left(C_{l}\right)=\sum_{n \geq l} \mu_{\lambda}\left(F_{\lambda}^{-1}\left(C_{n}\right) \cap \mathscr{B}_{r}\right) .
$$

In Corollary 3.10, that we admit for the moment, we show that there exists $K_{1}>0$, independent of $\lambda$, such that for any $A \subset B(0, r)$ we have

$$
\frac{1}{K_{1}} m_{\lambda}(A) \leq \mu_{\lambda}\left(F_{\lambda}^{-1}(A) \cap \mathscr{B}_{r}\right) \leq K_{1} m_{\lambda}(A) .
$$

So,

$$
\frac{1}{K_{1}} \sum_{n \geq l} m_{\lambda}\left(C_{n}\right) \leq \mu_{\lambda}\left(C_{l}\right) \leq K_{1} \sum_{n \geq l} m_{\lambda}\left(C_{n}\right) .
$$

From Proposition 3.6 we deduce that there exists $K_{2}>0$ such that

$$
\frac{1}{K_{2}} \sum_{n \geq l} \sum_{n \geq l} a_{n}(2 d(\lambda)) \leq \mu_{\lambda}\left(C_{l}\right) \leq K_{2} \sum_{n \geq l} a_{n}(2 d(\lambda)) .
$$

With the notations used in the appendix this is exactly

$$
\frac{1}{K_{2}} S_{l,+\infty}(2 d(\lambda)) \leq \mu_{\lambda}\left(C_{l}\right) \leq K_{2} S_{l,+\infty}(2 d(\lambda))
$$

We then use Corollary 5.10 to finish the proof.

Lemma 3.9. There exists $K>0$ such that for all $\lambda=1+\varepsilon$, with $\varepsilon>0$ small enough, all $r>0$ small enough, and all $M>0$ big enough we have,

$$
\frac{1}{K} \leq \rho_{\lambda} \leq K \text { on } \mathscr{B}_{r, M} \text {, and } \rho_{\lambda} \leq K \text { on } \mathscr{B}_{r} .
$$

From this Lemma and Lemma 3.7 we easily conclude this.

Corollary 3.10. There exists $K>0$ such that for all $\lambda=1+\varepsilon$, with $\varepsilon>0$ small enough, all $r>0$ small enough, and for any measurable set $A \subset B(0, r)$ we have

$$
\frac{1}{K} m_{\lambda}(A) \leq \mu_{\lambda}\left(F_{\lambda}^{-1}(A) \cap \mathscr{B}_{r}\right) \leq K m_{\lambda}(A) .
$$

Proof. Let $r>0$ and $\varepsilon>0$ be small enough so that the assertions of Lemmas 3.7 and 3.9 hold. Let $K>0$ coming from Lemma 3.9 be larger than $\max \left\{\beta, \alpha^{-1}\right\}$, both $\alpha$ and $\beta$ coming from Lemma 3.7. By Lemma 3.7 we know that for any $A \subset B(0, r)$ we have

$$
\frac{1}{K} m_{\lambda}\left(F_{\lambda}^{-1}(A) \cap \mathscr{B}_{r}\right) \leq m_{\lambda}(A) \leq K m_{\lambda}\left(F_{\lambda}^{-1}(A) \cap \mathscr{B}_{r}\right) .
$$

From the right hand side inequality in Lemma 3.9 we know that

$$
\mu_{\lambda}\left(F_{\lambda}^{-1}(A) \cap \mathscr{B}_{r}\right) \leq K m_{\lambda}\left(F_{\lambda}^{-1}(A) \cap \mathscr{B}_{r}\right) .
$$

These two inequalities give us

$$
\mu_{\lambda}\left(F_{\lambda}^{-1}(A) \cap \mathscr{B}_{r}\right) \leq K^{2} m_{\lambda}(A) .
$$


For the other inequality we first note that Lemma 3.7 also asserts that

$$
\frac{1}{K} m_{\lambda}\left(F_{\lambda}^{-1}(A) \cap \mathscr{B}_{r, M}\right) \leq m_{\lambda}\left(F_{\lambda}^{-1}(A) \cap \mathscr{B}_{r}\right) \leq K m_{\lambda}\left(F_{\lambda}^{-1}(A) \cap \mathscr{B}_{r, M}\right) .
$$

Since Lemma 3.9 implies that

$$
\frac{1}{K} m_{\lambda}\left(F_{\lambda}^{-1}(A) \cap \mathscr{B}_{r, M}\right) \leq \mu_{\lambda}\left(F_{\lambda}^{-1}(A) \cap \mathscr{B}_{r, M}\right) \leq K m_{\lambda}\left(F_{\lambda}^{-1}(A) \cap \mathscr{B}_{r, M}\right),
$$

we conclude that

$$
m_{\lambda}(A) \leq K m_{\lambda}\left(F_{\lambda}^{-1}(A) \cap \mathscr{B}_{r}\right) \leq K^{2} m_{\lambda}\left(F_{\lambda}^{-1}(A) \cap \mathscr{B}_{r, M}\right) \leq K^{3} \mu_{\lambda}\left(F_{\lambda}^{-1}(A) \cap \mathscr{B}_{r, M}\right) .
$$

We easily deduce that

$$
m_{\lambda}(A) \leq K^{3} \mu_{\lambda}\left(F_{\lambda}^{-1}(A) \cap \mathscr{B}_{r}\right) .
$$

This is the left hand side inequality of the Corollary and its proof is finished.

Proof. Before starting the proof of Lemma 3.9 we sketch the strategy. We first use a result of Urbański and Zdunik, Lemma 3.4 in $\left[\mathrm{Ur}, \mathrm{Zd}^{1}\right]$, that asserts that as long as we stay far away from the post-singular set, iterates of $\mathscr{L}_{\lambda}$ are uniformly bounded from above by a constant that does not depend on $\lambda$. This gives us that $\rho_{\lambda}$ is bounded from above in some $\mathscr{B}_{r}$. And this allows us to prove that for $r$ and $\varepsilon$ small enough, and for $M$ big enough we have

$$
\frac{1}{2} \leq \mu_{\lambda}\left(\mathscr{B}_{r, M}\right) \leq 1
$$

In order to control $\rho_{\lambda}$ on $\mathscr{B}_{r, M}$ we use Kobe's Distortion Theorem on $\mathscr{B}_{r, M}$ and prove that the measures $m_{\lambda}$ have the bounded distortion property on $\mathscr{B}_{r, M}$, with a constant which only depends on $r$ and $M$. This implies, see [Ma] (compare [Ha] Propositions 1.2 .7 and 1.2.8), that there exists an $F_{\lambda}$-invariant measure $\nu_{\lambda}$ which gives mass 1 to $\mathscr{B}_{r, M}$ and which is equivalent with $m_{\lambda}$. Its Radon-Nikodym derivative is such that $\frac{1}{K} \leq \frac{d \nu_{\lambda}}{d m_{\lambda}} \leq K$ on $\mathscr{B}_{r, M}$, with some $K>0$ independent of $\lambda$. Since $m_{\lambda}$ is ergodic and conservative, there is, up to a multiplicative constant, only one possible invariant measure equivalent to it. This means that $\mu_{\lambda}=\alpha_{\lambda} \nu_{\lambda}$. Integrating on $\mathscr{B}_{r, M}$ we conclude that $\alpha_{\lambda}=\mu_{\lambda}\left(\mathscr{B}_{r, M}\right)$. This leads to $\frac{1}{2 K} \leq \rho_{\lambda} \leq K$.

We now go into further details. Note that the singular set of $F_{\lambda}$ is the one point $-\lambda$ whose sequence of iterates converges towards 0 from the left. In particular, $\mathscr{B}_{r, M}$ is a simply connected domain on which inverse branches of $F_{\lambda}$ are well defined. Since $J_{\lambda}$ is a subset of $\left\{-\frac{\pi}{2} \leq \operatorname{Im} z \leq \frac{\pi}{2}\right\}$, one may find an open simply connected domain $U_{r, M}$ such that $\overline{U_{r, M}} \subset \mathscr{B}_{\frac{r}{2}, 2 M}$ and $J_{\lambda} \cap \mathscr{B}_{r, M} \subset U_{r, M}$. We have thus an annulus $\mathscr{B}_{\frac{r}{2}, 2 M} \backslash U_{r, M}$ and an associate Kobe constant $\sqrt{K_{r, M}}$. We conclude that for any $\lambda$ and any $n \in \mathbf{N}$ we have

$$
\forall x \in U_{r, M} \forall y \in U_{r, M} \quad \frac{1}{K_{r, M}} \leq \frac{\mathscr{L}_{\lambda}^{n}(1)(x)}{\mathscr{L}_{\lambda}^{n}(1)(y)} \leq K_{r, M} .
$$

Since for a measurable set $A$ we have $m_{\lambda}\left(F_{\lambda}^{-n}(A)\right)=\int_{A} \mathscr{L}_{\lambda}^{n}(1) d m_{\lambda}$, we conclude, if $A \subset U_{r, M}$, that

$$
\frac{1}{K_{r, M}} \frac{m_{\lambda}(A)}{m_{\lambda}\left(U_{r, M}\right)} \leq \frac{m_{\lambda}\left(F_{\lambda}^{-n}(A)\right)}{m_{\lambda}\left(F_{\lambda}^{-n}\left(U_{r, M}\right)\right)} \leq K_{r, M} \frac{m_{\lambda}(A)}{m_{\lambda}\left(U_{r, M}\right)}
$$

This is precisely the bounded distortion property for $m_{\lambda}$ on $U_{r, M}$ as it is used in [Ha]. Since $\left(J_{\lambda}, F_{\lambda}, m_{\lambda}\right)$ is ergodic and conservative there is, up to a multiplicative 
constant, only one invariant measure equivalent with $m_{\lambda}$. Let $\nu_{\lambda}$ be the one that gives mass 1 to $\mathscr{B}_{r, M}$. It follows from Propositions 1.2.7 and 1.2.8 in [Ha] that

$$
m_{\lambda^{-}} \text {almost surely on } \mathscr{B}_{r, M} \quad \frac{1}{K_{r, M}} \leq \frac{d \nu_{\lambda}}{d m_{\lambda}} \leq K_{r, M} \text {. }
$$

The measures $\mu_{\lambda}$ and $\nu_{\lambda}$ only differ by a multiplicative constant which can be computed by integrating the function 1 over $\mathscr{B}_{r, M}$. We deduce that $\mu_{\lambda}=\mu_{\lambda}\left(\mathscr{B}_{r, M}\right) \nu_{\lambda}$ and we conclude that

$$
m_{\lambda} \text {-almost surely on } \mathscr{B}_{r, M} \frac{\mu_{\lambda}\left(\mathscr{B}_{r, M}\right)}{K_{r, M}} \leq \rho_{\lambda} \leq K_{r, M} \mu_{\lambda}\left(\mathscr{B}_{r, M}\right) \text {. }
$$

Using inequalities (3.11) one may now adapt the reasoning of Lemma 3.4 in [Ur,Zd $\left.{ }^{1}\right]$ to our situation. Let $M$ be large enough and $r$ small enough so that $\frac{\log M-1}{2} \geq r$ and for all $\lambda \in\left[1, \lambda_{0}\right]$, if $\operatorname{Re} z>M$, then $\mathscr{L}_{\lambda}(1)(z) \leq 1$. The purpose of the first requirement is the following

$$
\left.\forall z \in P \quad\left(\operatorname{Re} z>M \text { and } F_{\lambda}(y)=z\right) \Rightarrow|y|>r \text { (i.e., } y \in \mathscr{B}_{r}\right) .
$$

We prove by induction that $H_{n}$ is true for all $n$ with

$$
H_{n} \Leftrightarrow\left\|\mathscr{L}_{\lambda}^{n}(1) \chi_{B_{r}}\right\|_{\infty} \leq \frac{K_{r, M}}{m_{\lambda}\left(\mathscr{B}_{r, M}\right)} .
$$

Notice that $H_{0}$ is obvious and assume that $H_{n}$ is true. Since

$$
\mathscr{L}_{\lambda}(1)(z) \leq \sum_{k \geq \operatorname{Re} z} \frac{2}{k^{d(\lambda)}},
$$

and since $d(\lambda)$ is converging towards $d\left(\lambda_{0}\right)$, one deduces that $\mathscr{L}_{\lambda}(1)(z)$ is, uniformly in $\lambda$, converging towards 0 as $\operatorname{Re} z \rightarrow \infty$. We deduce that $\left\|\mathscr{L}_{\lambda}(1) \chi_{\mathscr{B}_{r}}\right\|_{\infty}$ is achieved for some $z_{1} \in \mathscr{B}_{r}$. An easy induction leads, for all integers $n \geq 0$, to the existence of some $z_{n} \in \mathscr{B}_{r}$ such that

$$
\left\|\mathscr{L}_{\lambda}^{n}(1) \chi_{B_{r}}\right\|_{\infty}=\mathscr{L}_{\lambda}^{n}(1)\left(z_{n}\right) .
$$

Consider $z_{n+1}$ and assume that it lies in $\mathscr{B}_{r, M}$. Then we have

$$
1=\int \mathscr{L}_{\lambda}^{n+1}(1) d m_{\lambda} \geq \int \mathscr{L}_{\lambda}^{n+1}(1) \chi_{\mathscr{B}_{r, M}} d m_{\lambda} \geq \frac{\mathscr{L}_{\lambda}^{n+1}(1)\left(z_{n+1}\right)}{K_{r, M}} m_{\lambda}\left(\mathscr{B}_{r, M}\right) .
$$

The last inequality is an application of (3.11) and we conclude that $H_{n+1}$ is true. But $z_{n+1}$ might be with a real part greater than $M$. In this case we have

$$
\mathscr{L}_{\lambda}^{n+1}(1)\left(z_{n+1}\right)=\mathscr{L}_{\lambda}\left(\mathscr{L}_{\lambda}^{n}(1)\right)\left(z_{n+1}\right) \leq \mathscr{L}_{\lambda}^{n}(1)\left(z_{n}\right) \mathscr{L}_{\lambda}(1)\left(z_{n+1}\right) \leq \mathscr{L}_{\lambda}^{n}\left(z_{n}\right) .
$$

Those inequalities are implied by our assumptions on $M$ and $r$ that ensure us first, that any pre-image of $z_{n+1}$ is in $\mathscr{B}_{r}$, and second, that $\mathscr{L}_{\lambda}(1)\left(z_{n+1}\right) \leq 1$. We may now apply our inductive assumption to conclude that $H_{n+1}$ is true so that this hypothesis is true for any integer $n$. Let $\alpha_{r, M, \lambda_{0}}$ be defined as the infimum of the set $\left(m_{\lambda}\left(\mathscr{B}_{r, M}\right)\right)$ where $\lambda \in\left[1, \lambda_{0}\right]$. Since $\lambda \mapsto m_{\lambda}\left(\mathscr{B}_{r, M}\right)$ is continuous on $\left[1, \lambda_{0}\right]$, this infimum is achieved and is strictly greater than 0 . Fix $r$ small and choose $M(r)$ such that all assumptions are fulfilled and set $C_{r, \lambda_{0}}=\frac{K_{r, M(r)}}{\alpha_{r, M(r), \lambda_{0}}}$. We deduce from our analysis that $\lim _{n \rightarrow \infty} \mathscr{L}_{\lambda}^{n}(1)=\rho_{\lambda} \leq C_{r, \lambda_{0}}$ on $\mathscr{B}_{r}$. We have thus proved the left hand side inequality of Lemma 3.9. In order to finish the proof of this Lemma we need to 
prove that $\frac{1}{K} \leq \rho_{\lambda} \leq K$ on $\mathscr{B}_{r, M}$. By (3.12) this will be done if one can prove that $\mu_{\lambda}\left(\mathscr{B}_{r, M}\right) \geq \frac{1}{2}$ for suitable $r$ and $M$.

Since we know that $\rho_{\lambda} \leq C_{r, \lambda_{0}}$ on $\mathscr{B}_{r}$, we may already use the left-hand side inequalities of Proposition 3.8. In particular, for any $n$ we have

$$
\mu_{\lambda}\left(C_{n}\right) \leq \frac{C_{r, \lambda_{0}}}{n^{2 \delta-1}} \quad \text { with } 1<\delta=\inf \{d(\lambda)\}, \text { well defined by continuity. }
$$

Let now $N$ be big enough so that

$$
\sum_{n \geq N} \frac{1}{n^{2 \delta-1}} \leq \frac{1}{4 C_{r, \lambda_{0}}}
$$

Choose $r^{\prime}$ small enough so that for any $\lambda \in\left[1, \lambda_{0}\right]$ we have

$$
B\left(0, r^{\prime}\right) \subset \cup_{n \geq N} C_{n}(\lambda) .
$$

Such a choice is possible because of Proposition 5.7. We then easily conclude that $\mu_{\lambda}\left(B\left(0, r^{\prime}\right)\right) \leq \frac{1}{4}$. As a consequence, one may assume, without loss of generality, that we have started our analysis with $r>0$ small enough so that $\mu_{\lambda}(B(0, r)) \leq \frac{1}{4}$.

By Lemma 4.1 in $\left[\mathrm{Ur}, \mathrm{Zd}^{2}\right]$, we know that the sequence of measures $\left(m_{\lambda}\right)$ is tight. In particular, if $M$ is chosen large enough, then for any $\lambda \in\left[1, \lambda_{0}\right]$ we have $m_{\lambda}\left(P_{M}^{c}\right) \leq$ $\frac{1}{4 C_{r, \lambda_{0}}}$. From where we deduce that $\mu_{\lambda}\left(P_{M}^{c}\right) \leq \frac{1}{4}$.

Note now that $\mu_{\lambda}\left(\mathscr{B}_{r, M}\right)=1-\mu_{\lambda}(B(0, r))-\mu_{\lambda}\left(P_{M}^{c}\right) \geq \frac{1}{2}$. As already mentioned, this inequality finishes the proof of the lemma.

\section{Controlling the integrals}

In this section we mainly reproduce the reasoning of $\left[\mathrm{Ha}, \mathrm{Zi}^{1}\right]$. Nevertheless there are some differences we would like to emphasize: the main being that we do not know whether the dimension of $J\left(F_{1}\right)$ is less than $\frac{3}{2}$ or not. Note also that the Markov partition used in $\left[\mathrm{Ha}, \mathrm{Zi}^{1}\right]$ is replaced in the present article by the backward images of the fundamental domain $C_{0}$. Finally, note that we work directly on $J_{\lambda}$ without conjugating the dynamics.

Before we start the proofs and in order to simplify some expressions and calculations, we introduce the following notation. Let

$$
\Psi_{n}=\sum_{k=1}^{n} \frac{1}{\left(F_{\lambda}^{k}\right)^{\prime}}, \quad \Phi_{n}=\sum_{k=1}^{n} \frac{1}{\left|\left(F_{\lambda}^{k}\right)^{\prime}\right|}, \quad \Psi=\sum_{k=1}^{\infty} \frac{1}{\left(F_{\lambda}^{k}\right)^{\prime}} \quad \text { and } \quad \Phi=\sum_{k=1}^{+\infty} \frac{1}{\left|\left(F_{\lambda}^{k}\right)^{\prime}\right|}
$$

so that formula (2.1) may be written

$$
d^{\prime}(\lambda)=-\frac{d(\lambda)}{\chi_{\mu_{\lambda}}}\left(1-\frac{1}{\lambda}\right) \int_{J_{\lambda}} \operatorname{Re}(\Psi) d \mu_{\lambda} .
$$

We will need the following equation which is an easy computation

$$
\Psi=\frac{1}{\left(F_{\lambda}^{n}\right)^{\prime}} \Psi \circ F_{\lambda}^{n}+\Psi_{n}, \quad \Phi=\frac{1}{\left|\left(F_{\lambda}^{n}\right)^{\prime}\right|} \Phi \circ F_{\lambda}^{n}+\Phi_{n} .
$$

4.1. Lyapunov exponents. In this paragraph we prove that the Lyapunov exponents do not play any role in our estimates of the derivative. In order to do this we only need to check that they are uniformly bounded above and separated away from zero. More precisely we prove the following. 
Proposition 4.1. There exist $r_{0}>0, \lambda_{0}>1$ and $K>1$ such that $\forall \lambda \in\left(\lambda, \lambda_{0}\right)$ we have

$$
\frac{1}{K} \leq \chi_{\mu_{\lambda}}:=\int_{J_{\lambda}} \log \left|F_{\lambda}^{\prime}\right| d \mu_{\lambda} \leq K
$$

Proof. First note that $\forall \lambda \geq 1$ and $\forall z \in J_{\lambda}$ we have $\left|F_{\lambda}^{\prime}(z)\right| \geq 1$. In particular, we have

$$
\int_{C_{0}} \log \left|F_{\lambda}^{\prime}\right| d \mu_{\lambda} \leq \chi_{\mu_{\lambda}}
$$

There is $K_{1}>0$ such that $\operatorname{Re} z \geq K_{1}$ for any $z \in C_{0}(\lambda)$ and any $\lambda \in\left(1, \lambda_{0}\right)$, and by Proposition 5.5 there is $K_{2}$ such that $\mu_{\lambda}\left(C_{0}\right) \geq K_{2}$. Since $\log \left|F_{\lambda}^{\prime}(z)\right|=\log \lambda+\operatorname{Re} z$, we deduce that

$$
0<K_{1} K_{2} \leq \int_{C_{0}} \log \left|F_{\lambda}^{\prime}\right| d \mu_{\lambda} \leq \chi_{\mu_{\lambda}}
$$

This is the first part of the proof.

For the other part note first that continuity of $\lambda \mapsto d(\lambda)$ and the fact that $d(1)>1$ imply that there exist $\alpha>1$ and $\beta>0$ such that $\alpha+\beta \leq d(\lambda)$ for any $\lambda \in\left(1, \lambda_{0}\right)$. This implies, in particular, that $\forall \lambda \in\left(1, \lambda_{0}\right)$ and $\forall z \in J_{\lambda}$

$$
\frac{1}{\left|\left(F_{\lambda}\right)^{\prime}(z)\right|^{d(\lambda)}} \leq \frac{1}{\left|\left(F_{\lambda}\right)^{\prime}(z)\right|^{\alpha+\beta}} \text {. }
$$

Consider now the following partition of the strip $P:\left(A_{n}\right)_{n \in \mathbf{N}}$, with $A_{n}:=\{z \in P \mid$ $n-1<\operatorname{Re} z \leq n\}$. We have

$$
\chi_{\mu_{\lambda}}=\sum_{n=1}^{+\infty} \int_{A_{n}} \log \left|F_{\lambda}^{\prime}\right| d \mu_{\lambda} \leq \log \lambda_{0}+\sum_{n=1}^{+\infty} \int_{A_{n}} \operatorname{Re} z d \mu_{\lambda}(z) \leq \log \lambda_{0}+\sum_{n=1}^{+\infty} n \mu_{\lambda}\left(A_{n}\right) .
$$

Lemma 3.9 implies that there exists $K_{3}>0$ such that $\mu_{\lambda}\left(A_{n}\right) \leq K_{3} m_{\lambda}\left(A_{n}\right)$ for $n \geq 2$. Note now that

$$
m_{\lambda}\left(A_{n}\right)=\int_{J_{\lambda}} \chi_{A_{n}} d m_{\lambda}=\int_{J_{\lambda}} \mathscr{L}_{\lambda}\left(\chi_{A_{n}}\right) d m_{\lambda} .
$$

For any $z \in J_{\lambda}$ and any $k \in \mathbf{Z}$ we let $z_{k}$ be the preimage of $z$ for $F_{\lambda}$ such that $f_{\lambda}\left(z_{k}\right)=z+2 i k \pi$. We thus have

$$
\mathscr{L}_{\lambda}\left(\chi_{A_{n}}\right)(z)=\sum_{k \in \mathbf{Z}} \frac{1}{\left|F_{\lambda}^{\prime}\left(z_{k}\right)\right|^{d(\lambda)}} \chi_{A_{n}}\left(z_{k}\right) .
$$

With $\alpha$ and $\beta$ defined above, this gives that

$$
\mathscr{L}_{\lambda}\left(\chi_{A_{n}}\right)(z) \leq \sum_{k \in \mathbf{Z}} \frac{1}{\left|F_{\lambda}^{\prime}\left(z_{k}\right)\right|^{\alpha+\beta}} \chi_{A_{n}}\left(z_{k}\right) .
$$

Since $\left|F_{\lambda}^{\prime}\left(z_{k}\right)\right|=\lambda e^{\operatorname{Re} z_{k}}=|z+\lambda+2 i k \pi|$, we have

$$
\frac{1}{\left|F_{\lambda}^{\prime}\left(z_{k}\right)\right|^{\alpha+\beta}} \chi_{A_{n}}\left(z_{k}\right) \leq \frac{1}{|z+\lambda+2 i k \pi|^{\alpha}} \lambda^{-\beta} e^{-\beta(n-1)} \text {, }
$$

so that

$$
\mathscr{L}_{\lambda}\left(\chi_{A_{n}}\right)(z) \leq \lambda^{-\beta} e^{-\beta n} \sum_{k \in \mathbf{Z}} \frac{1}{|z+\lambda+2 i k \pi|^{\alpha}}
$$


As we have $\alpha>1$, there is $K_{4}>0$, independent of $\lambda$ and $z$, such that

$$
\sum_{k \in \mathbf{Z}} \frac{1}{|z+\lambda+2 i k \pi|^{\alpha}}<K_{4} \lambda^{\beta} \text {. }
$$

This tells us that

$$
\mathscr{L}_{\lambda}\left(\chi_{A_{n}}\right)(z) \leq K_{4} e^{-\beta n}
$$

Integrating with respect to $m_{\lambda}$, and summing over $n \geq 2$, we get

$$
\chi_{\mu_{\lambda}} \leq \log \lambda_{0}+K_{3} m_{\lambda}\left(A_{1}\right)+K_{3} K_{4} \sum_{n \geq 2} e^{-\beta n} \leq K_{5},
$$

with $K_{5}:=\log \lambda_{0}+K_{3}+K_{3} K_{4} \frac{e^{-2 \beta}}{1-e^{-\beta}}$. This is clearly independent of $\lambda$ and we are done.

Note that with some more work one can indeed prove that $\chi_{\mu_{\lambda}}$ converges towards $\chi_{\mu_{1}}$ as $\lambda$ converges towards 1 from above.

4.2. Controlling the integral away from 0 . Let $N$ be an integer ${ }^{6}$ and set $M_{N}=\bigcup_{n \geq N+1} C_{n}$ and $B_{N}=J_{\lambda} \backslash M_{N}$. Note that both sets $M_{N}$ and $B_{N}$ depend on $\lambda$.

Proposition 4.2. There exists $k(N)>0$ such that $\forall \lambda \in\left[1, \lambda_{0}\right]$ we have

$$
\int_{B_{N}} \Phi d \mu_{\lambda} \leq k(N)
$$

Proof. Let $D_{0}=B_{N}$, and for any $n \in \mathbf{N}$ let $D_{n}=C_{N+n}$. Following [Ha,Zi $\left.{ }^{1}\right]$, let $U_{n}$ be the set of points which arrive or come back to $B_{N}$ after exactly $n$ iterates, which means that $U_{n}=F_{\lambda}^{-1}\left(D_{n-1}\right)$. Note that $U_{n} \cap M_{n}=D_{n}$. Given $N_{0} \in \mathbf{N}$ we set $A_{n}=F_{\lambda}^{-N_{0}}\left(U_{n}\right) \cap B_{N}$. Since $\left(U_{n}\right)$ is a partition of $J_{\lambda}$, and $\left(A_{n}\right)$ is a partition of $B_{N}$, we have

$$
\int_{B_{N}} \Phi d \mu_{\lambda}=\sum_{k=1}^{+\infty} \int_{A_{k}} \Phi d \mu_{\lambda}
$$

Using relation 4.1 with $n=N_{0}+k$, we get

$$
\int_{A_{k}} \Phi d \mu_{\lambda}=\int_{A_{k}}\left(\frac{1}{\left|\left(F_{\lambda}^{N_{0}+k}\right)^{\prime}\right|} \Phi \circ F_{\lambda}^{N_{0}+k}+\Phi_{N_{0}+k}\right) d \mu_{\lambda}
$$

Using the fact that $F_{\lambda}^{N_{0}+k}\left(A_{k}\right) \subset B_{N}$, Lemma 3.5 and Lemma 3.4, we deduce that

$$
\int_{A_{k}} \Phi d \mu_{\lambda} \leq \frac{\kappa(N)}{\left(N_{0}+k\right)^{2}} \int_{A_{k}} \Phi \circ F_{\lambda}^{N_{0}+k} d \mu_{\lambda}+\left(N_{0}+k\right) \mu_{\lambda}\left(A_{k}\right) .
$$

The fact that $F_{\lambda}^{N_{0}+k}\left(A_{k}\right) \subset B_{N}$ also implies that $\chi_{A_{k}} \leq \chi_{B_{N}} \circ F_{\lambda}^{N_{0}+k}$, from the invariance of $\mu_{\lambda}$ we thus get

$$
\int_{A_{k}} \Phi \circ F_{\lambda}^{N_{0}+k} d \mu_{\lambda} \leq \int \chi_{B_{N}} \circ F_{\lambda}^{N_{0}+k} \Phi \circ F_{\lambda}^{N_{0}+k} d \mu_{\lambda} \leq \int_{B_{N}} \Phi d \mu_{\lambda},
$$

which leads to

$$
\int_{A_{k}} \Phi d \mu_{\lambda} \leq \frac{\kappa(N)}{\left(N_{0}+k\right)^{2}} \int_{B_{N}} \Phi d \mu_{\lambda}+\left(N_{0}+k\right) \mu_{\lambda}\left(A_{k}\right) .
$$

\footnotetext{
${ }^{6}$ This integer will be chosen later big enough to ensure that for any $z_{n} \in C_{n}$ we have $\sum_{n \geq N} \arg \left(z_{n}\right) \leq \frac{\pi}{6}$.
} 
In order to estimate $\mu_{\lambda}\left(A_{k}\right)$, we first use Lemma 3.9 to conclude that $\mu_{\lambda}\left(A_{k}\right) \leq$ $K m_{\lambda}\left(A_{k}\right) \leq K m_{\lambda}\left(F_{\lambda}^{-N_{0}}\left(U_{k}\right)\right)$ for some constant $K$ independent of $k, N_{0}$ and $\lambda$. Since $U_{k}=F_{\lambda}^{-1}\left(D_{k}\right)$, we get $\mu_{\lambda}\left(A_{k}\right) \leq K m_{\lambda}\left(F_{\lambda}^{-\left(N_{0}+1\right)}\left(D_{k}\right)\right)$. Moreover,

$$
m_{\lambda}\left(F_{\lambda}^{-\left(N_{0}+1\right)}\left(D_{k}\right)\right)=\int \chi_{D_{k}} \circ F_{\lambda}^{N_{0}+1} d m_{\lambda}=\int_{D_{k}} \mathscr{L}_{\lambda}^{N_{0}+1}(1) d m_{\lambda},
$$

since there exists $K_{1}\left(N_{0}\right)$ independent of $\lambda$ and $k$ such that $\mathscr{L}_{\lambda}^{N_{0}+1}(1) \leq K_{1}\left(N_{0}\right)$, using Lemma 3.6 and the fact that $D_{k}=C_{N+k}$, we get

$$
m_{\lambda}\left(F_{\lambda}^{-\left(N_{0}+1\right)}\left(D_{k}\right) \leq K_{1}\left(N_{0}\right) m_{\lambda}\left(C_{N+k}\right) \leq \frac{K_{2}}{(N+k)^{2 d(\lambda)}} .\right.
$$

Using the fact that $\left(N_{0}+k\right) \leq N_{0}(N+k)$, we thus conclude that

$$
\int_{A_{k}} \Phi d \mu_{\lambda} \leq \frac{\kappa(N)}{\left(N_{0}+k\right)^{2}} \int_{B_{N}} \Phi d \mu_{\lambda}+\frac{K K_{2} N_{0}}{(N+k)^{2 d(\lambda)-1}} .
$$

Summing over $k$ we end up with

$$
\int_{B_{N}} \Phi d \mu_{\lambda} \leq \frac{\kappa(N)}{N_{0}-1} \int_{B_{N}} \Phi d \mu_{\lambda}+\frac{K K_{2} N_{0}}{(N-1)^{2 d(\lambda)-2}}
$$

The integer $N$ being fixed, one may now choose $N_{0}$ big enough so that $\frac{\kappa(N)}{N_{0}-1} \leq \frac{1}{2}$. Hence

$$
\int_{B_{N}} \Phi d \mu_{\lambda} \leq \frac{2 K K_{2} N_{0}}{(N-1)^{2 d(\lambda)-2}} .
$$

This last constant depends only on $N$ and we are done.

4.3. Controlling the integral in a neighborhood of 0 . In this paragraph we deal with the remaining part of $\int \operatorname{Re}(\Psi) d \mu_{\lambda}$. Putting $M_{N}=J_{\lambda} \backslash B_{N}$, we prove the following

Proposition 4.3. There exists $K>0$ and $N \in \mathbf{N}$ such that for $\forall \lambda \in\left(1, \lambda_{0}\right)$,

$$
\begin{aligned}
\frac{1}{K}(\lambda-1)^{2 d(\lambda)-3} & \leq \int_{M_{N}} \operatorname{Re}(\Psi) d \mu_{\lambda} \leq K(\lambda-1)^{2 d(\lambda)-3} \text { if } d(\lambda)<\frac{3}{2}, \\
-\frac{1}{K} \log (\lambda-1) & \leq \int_{M_{N}} \operatorname{Re}(\Psi) d \mu_{\lambda} \leq-K \log (\lambda-1) \text { if } d(\lambda)=\frac{3}{2}, \\
\left|\int_{M_{N}} \operatorname{Re}(\Psi) d \mu_{\lambda}\right| & \leq K \text { if } d(\lambda)>\frac{3}{2} .
\end{aligned}
$$

Proof. We split this integral into several pieces. First we note using (4.1) that

$$
\int_{M_{N}} \operatorname{Re}(\Psi) d \mu_{\lambda}=\sum_{n=N+1}^{+\infty}\left[\int_{C_{n}} \operatorname{Re}\left(\frac{1}{\left(F_{\lambda}^{n-N}\right)^{\prime}} \Psi \circ F_{\lambda}^{n-N}\right) d \mu_{\lambda}+\int_{C_{n}} \operatorname{Re}\left(\Psi_{n-N}\right) d \mu_{\lambda}\right] .
$$

We first deal with the left hand side of the sum that we bound integrating the modulus of the function,

$$
\left|\int_{C_{n}} \operatorname{Re}\left(\frac{1}{\left(F_{\lambda}^{n-N}\right)^{\prime}} \Psi \circ F_{\lambda}^{n-N}\right) d \mu_{\lambda}\right| \leq \int_{C_{n}} \frac{1}{\left|\left(F_{\lambda}^{n-N}\right)^{\prime}\right|} \Phi \circ F_{\lambda}^{n-N} d \mu_{\lambda} .
$$


We use Lemma 3.5 and the fact that for $z \in C_{n}$, we have $F_{\lambda}^{n-N}(z) \in C_{N} \subset B_{N}$ to conclude that

$$
\left|\int_{C_{n}} \operatorname{Re}\left(\frac{1}{\left(F_{\lambda}^{n-N}\right)^{\prime}} \Psi \circ F_{\lambda}^{n-N}\right) d \mu_{\lambda}\right| \leq \frac{K}{(n-N)^{2}} \int_{B_{N}} \Phi d \mu_{\lambda} .
$$

Summing over $n \geq N$ we get

$$
\left|\sum_{n=N+1}^{+\infty}\left[\int_{C_{n}} \operatorname{Re}\left(\frac{1}{\left(F_{\lambda}^{n-N}\right)^{\prime}} \Psi \circ F_{\lambda}^{n-N}\right) d \mu_{\lambda}\right]\right| \leq K \int_{B_{N}} \Phi d \mu_{\lambda} \sum_{n=1}^{+\infty} \frac{1}{n^{2}} .
$$

By Proposition 4.2 we conclude that there exists $K(N)>0$ such that

$$
\left|\sum_{n=N}^{+\infty}\left[\int_{C_{n}} \operatorname{Re}\left(\frac{1}{\left(F_{\lambda}^{n-N}\right)^{\prime}} \Psi \circ F_{\lambda}^{n-N}\right) d \mu_{\lambda}\right]\right| \leq K(N) .
$$

We now deal with the right hand side. We have

$$
\int_{C_{n}} \operatorname{Re}\left(\Psi_{n-N}\right) d \mu_{\lambda}=\sum_{k=1}^{n-N} \int_{C_{n}} \operatorname{Re}\left(\frac{1}{\left(F_{\lambda}^{k}\right)^{\prime}}\right) d \mu_{\lambda} .
$$

Choose $N$ big enough so that conclusions of Lemma 3.2 hold. For any $z \in C_{n}$ and any $k \leq n-N$, we have

so that

$$
\frac{\sqrt{3}}{2}\left|F_{\lambda}^{k}(z)\right| \leq \operatorname{Re}\left(F_{\lambda}^{k}\right)^{\prime}(z)
$$

$$
\int_{C_{n}} \operatorname{Re}\left(\psi_{n-N}\right) d \mu_{\lambda} \sim \sum_{k=1}^{n-N} \int_{C_{n}} \operatorname{Re}\left(\frac{1}{\left|F_{\lambda}^{k}\right|^{\prime}}\right) d \mu_{\lambda} .
$$

Note now that for any $z \in C_{n}$, we have by the Chain Rule that

$$
\left(F_{\lambda}^{k}\right)^{\prime}(z)=\frac{\left(F_{\lambda}^{n}\right)^{\prime}(z)}{\left(F_{\lambda}^{n-k}\right)^{\prime}\left(F_{\lambda}^{k}(z)\right)},
$$

with $F_{\lambda}^{k}(z) \in C_{n-k}$. We deduce, using Proposition 3.1, that

$$
\frac{1}{\left|\left(F_{\lambda}^{k}\right)^{\prime}(z)\right|} \sim \frac{a_{n}(2)}{a_{n-k}(2)}
$$

Estimates of $\mu_{\lambda}\left(C_{n}\right)$ are given by Proposition 3.8, and we conclude that

$$
\int_{C_{n}} \operatorname{Re}\left(\psi_{n-N}\right) d \mu_{\lambda} \sim \begin{cases}a_{n}(2 d(\lambda)-1) a_{n}(2) \sum_{k=1}^{n-N} a_{n-k}(-2) & \text { if } n \leq N_{\varepsilon}, \\ \frac{1}{\varepsilon} a_{n}(2 d(\lambda)) a_{n}(2) \sum_{k=1}^{n-N} a_{n-k}(-2) & \text { if } n \geq N_{\varepsilon} .\end{cases}
$$

Since $a_{n}(\alpha) a_{n}(\beta)=a_{n}(\alpha+\beta)$, and with $S_{k, n}(\alpha)=\sum_{k}^{n} a_{j}(\alpha)$, this can also be written

$$
\int_{C_{n}} \operatorname{Re}\left(\psi_{n-N}\right) d \mu_{\lambda} \sim \begin{cases}a_{n}(2 d(\lambda)+1) S_{N, n-1}(-2) & \text { if } n \leq N_{\varepsilon}, \\ \frac{1}{\varepsilon} a_{n}(2 d(\lambda)+2) S_{N, n-1}(-2) & \text { if } n \geq N_{\varepsilon} .\end{cases}
$$

Using now Corollary 5.11, we have $S_{N, n-1}(-2) \sim\left(a_{n}(-3)-a_{N}(-3)\right)$ if $n \leq N_{\varepsilon}$, and $S_{N, n-1}(-2) \sim \frac{a_{n}(-2)}{\varepsilon}$ if $n \geq N_{\varepsilon}$, and we get

$$
\int_{C_{n}} \operatorname{Re}\left(\psi_{n-N}\right) d \mu_{\lambda} \sim \begin{cases}a_{n}(2 d(\lambda)+1)\left(a_{n}(-3)-a_{N}(-3)\right) & \text { if } n \leq N_{\varepsilon} \\ \frac{1}{\varepsilon^{2}} a_{n}(2 d(\lambda)+2) a_{n}(-2) & \text { if } n \geq N_{\varepsilon} .\end{cases}
$$


Since $a_{n}(\alpha) a_{n}(\beta)=a_{n}(\alpha+\beta)$, we get

$$
\int_{C_{n}} \operatorname{Re}\left(\psi_{n-N}\right) d \mu_{\lambda} \sim \begin{cases}a_{n}(2 d(\lambda)-2)-a_{N}(-3) a_{n}(2 d(\lambda)+1) & \text { if } n \leq N_{\varepsilon} \\ \frac{1}{\varepsilon^{2}} a_{n}(2 d(\lambda)) & \text { if } n \geq N_{\varepsilon}\end{cases}
$$

Summing over $n \geq N$ this gives us that $\sum_{n \geq N} \int_{C_{n}} \operatorname{Re}\left(\psi_{n-N}\right) d \mu_{\lambda}$ is comparable with

$$
\max \left(\left(S_{N, N_{\varepsilon}}(2 d(\lambda)-2)-a_{N}(-3) S_{N, N_{\varepsilon}}(2 d(\lambda)+1)\right), \frac{1}{\varepsilon^{2}} S_{N_{\varepsilon},+\infty}(2 d(\lambda))\right) .
$$

We then deduce from Corollary 5.10 and Corollary 5.11 that $S_{N, N_{\varepsilon}}(2 d(\lambda)+1) \sim$ $a_{N}(2 d(\lambda)) \sim 1$, and also that $S_{N_{\varepsilon},+\infty}(2 d(\lambda)) \sim \frac{a_{N \varepsilon}(2 d(\lambda))}{\varepsilon} \sim \varepsilon^{2 d(\lambda)-1}$. Estimates of $S_{N, N_{\varepsilon}}(2 d(\lambda)-2)$ depend on the comparison of $d(\lambda)$ with $\frac{3}{2}$. More precisely, if $d(\lambda)>\frac{3}{2}$, then Corollary 5.11 tells us that $S_{N, N_{\varepsilon}}(2 d(\lambda)-2) \sim 1$, if $d(\lambda)=\frac{3}{2}$, then it tells us that $S_{N, N_{\varepsilon}}(2 d(\lambda)-2) \sim \log N_{\varepsilon}$, and if $d(\lambda)<\frac{3}{2}$, then $S_{N, N_{\varepsilon}}(2 d(\lambda)-2) \sim \varepsilon^{2 d(\lambda)-3}$. Summarizing all those estimates we get

$$
\sum_{n \geq N} \int_{C_{n}} \operatorname{Re}\left(\psi_{n-N}\right) d \mu_{\lambda} \sim \begin{cases}1 & \text { if } d(\lambda)>\frac{3}{2} \\ \log N_{\varepsilon} & \text { if } d(\lambda)=\frac{3}{2} \\ \varepsilon^{2 d(\lambda)-3} & \text { if } d(\lambda)<\frac{3}{2}\end{cases}
$$

4.4. Proof of the main result. We are now in position to prove the main result of this paper that we recall here.

Theorem 4.4. There exists $\lambda_{0}>1$, and $K>1$ such that

$$
\begin{cases}\frac{-1}{K}(\lambda-1)^{2 d(1)-2} \leq d^{\prime}(\lambda) \leq-K(\lambda-1)^{2 d(1)-2} & \text { if } d(1)<\frac{3}{2} \\ \left|d^{\prime}(\lambda)\right| \leq K(\lambda-1) \log \frac{1}{\lambda-1} & \text { if } d(1)=\frac{3}{2} \\ \left|d^{\prime}(\lambda)\right| \leq K(\lambda-1) & \text { if } d(1)>\frac{3}{2}\end{cases}
$$

In particular, the function $\lambda \mapsto d(\lambda)$ is $C^{1}$ on $[1,+\infty)$, with $d^{\prime}(1)=0$.

Proof. Let us recall that we have

$$
d^{\prime}(\lambda)=-\frac{d(\lambda)}{\chi_{\mu_{\lambda}}}\left(1-\frac{1}{\lambda}\right) \int_{J_{\lambda}} \operatorname{Re} \Psi d \mu_{\lambda} .
$$

We first use $\left[\mathrm{Ur}, \mathrm{Zd}^{2}\right]$, where it is proved that $\lambda \mapsto d(\lambda)$ is continuous on $[1,+\infty)$, and Proposition 4.1 to conclude that there exists $\lambda_{1}>1$ and $K_{1}>1$ such that $\forall \lambda \in\left(1, \lambda_{1}\right)$ we have

$$
\frac{1}{K_{1}}(\lambda-1) \leq \frac{d(\lambda)}{\chi_{\mu_{\lambda}}}\left(1-\frac{1}{\lambda}\right) \leq K_{1}(\lambda-1) .
$$

Note that given any integer $N$, we have

$$
\int_{J_{\lambda}} \operatorname{Re} \Psi d \mu_{\lambda}=\int_{B_{N}} \operatorname{Re} \Psi d \mu_{\lambda}+\int_{M_{N}} \operatorname{Re} \Psi d \mu_{\lambda}
$$

so that

$$
\left|d^{\prime}(\lambda)\right| \leq 2 K_{1}(\lambda-1) \max \left(\left|\int_{B_{N}} \operatorname{Re} \Psi d \mu_{\lambda}\right|,\left|\int_{M_{N}} \operatorname{Re} \Psi d \mu_{\lambda}\right|\right) .
$$


We may thus use Proposition 4.2 and Proposition 4.3 to conclude that $d^{\prime}(\lambda)$ is converging towards 0 when $\lambda$ is converging towards 0 from above. In particular, there is $\lambda_{2}>1$ such that $\forall \lambda \in\left[1, \lambda_{2}\right)$,

We deduce that

$$
-\frac{1}{2} \leq d^{\prime}(\lambda) \leq \frac{1}{2}
$$

so that

$$
-\frac{1}{2}(\lambda-1) \leq d(\lambda)-d(1) \leq \frac{1}{2}(\lambda-1)
$$

$$
\begin{aligned}
(\lambda-1)^{\lambda-1}(\lambda-1)^{2 d(1)-3} & \leq(\lambda-1)^{2 d(\lambda)-3}=(\lambda-1)^{2 d(1)-3}(\lambda-1)^{2(d(\lambda)-d(1))} \\
& \leq(\lambda-1)^{-(\lambda-1)}(\lambda-1)^{2 d(1)-3}
\end{aligned}
$$

Since $\lambda \mapsto(\lambda-1)^{\lambda-1}$ is continuous on $\left[1, \lambda_{2}\right]$, there exists $K_{3}>1$ such that

$$
\frac{1}{K_{3}}(\lambda-1)^{2 d(1)-3} \leq(\lambda-1)^{2 d(\lambda)-3} \leq K_{3}(\lambda-1)^{2 d(1)-3} .
$$

Using again Proposition 4.2 and Proposition 4.3, and the fact we just proved that allows us to replace $d(\lambda)$ with $d(1)$, we conclude the proof of the main result in case $d(1)<\frac{3}{2}$.

In case $d(1)=\frac{3}{2}$, Propositions 4.2 and 4.3 tells us that the maximum in (4.3) is dominated by $-\log (\lambda-1)$. In case $d(1)>\frac{3}{2}$, the same proposition leads to the fact that this maximum is bounded.

\section{Appendices}

5.1. Estimates close to a repelling/parabolic fixed point. In this appendix we show how to get estimates in case of a degeneracy towards a multi-petal parabolic fixed point. It is a two steps proof: first we deal with the real axis then we extend estimates obtained in the real line to the complex plane using Kobe's Distortion Theorem.

Consider the following family of germs of holomorphic functions defined in a neighborhood of 0 that we denote by $\mathscr{U}$ :

$$
f_{\varepsilon}(z)=(1+\varepsilon) z+z^{p+1}+z^{p+2} g_{\varepsilon}(z) .
$$

Assume that there is an inverse branch $f_{\varepsilon}^{-1}$ well defined on $\mathscr{U}$ that leaves a sector $S_{\theta}:=\left\{r e^{i \alpha} \mid-\theta \leq \alpha \leq \theta\right\}$ invariant, for some $0<\theta<\frac{\pi}{2}$. Let $\mathscr{U}_{\theta}:=\mathscr{U} \cap S_{\theta}$. Assume also that $\forall z \in \mathscr{U}$ we have $\left|z g_{\varepsilon}(z)\right|<\frac{1}{2}$. Let $I=\mathscr{U} \cap \mathbf{R}^{+}$and assume that $f_{\varepsilon}^{-1}(I) \subset I$ and that $f_{\varepsilon}$ is not decreasing on $I$.

This appendix is organized as follows: in the first two paragraphs we study those germs giving in the second paragraph uniform estimates for $\left|\left(f_{\varepsilon}^{-n}\right)^{\prime}\right|$.

5.1.1. The Mean Value Theorem and its consequences. We start with the following easy fact.

Lemma 5.1. Let $f: \mathbf{R} \rightarrow \mathbf{R}^{+}$be a decreasing map with antiderivative $F$ on $\mathbf{R}$ and let $\left(u_{n}\right)_{n \in \mathbf{N}}$ be a decreasing sequence of real numbers. Suppose that there exist $n>1$ such that for all $k \leq n$ we have

(i) if $K_{1} \leq\left(u_{k}-u_{k+1}\right) f\left(u_{k}\right)$, then $K_{1} k \leq F\left(u_{0}\right)-F\left(u_{k}\right)$,

(ii) if $\left(u_{k}-u_{k+1}\right) f\left(u_{k+1}\right) \leq K_{2}$, then $F\left(u_{0}\right)-F\left(u_{k}\right) \leq K_{2} k$. 
Proof. One only needs to check that our assumptions imply

$$
\left(u_{k}-u_{k+1}\right) f\left(u_{k}\right) \leq \int_{u_{k+1}}^{u_{k}} f(t) d t \leq\left(u_{k}-u_{k+1}\right) f\left(u_{k+1}\right) .
$$

In particular we single out the following two particular cases:

Corollary 5.2. Let $\left(x_{n}\right)$ be a decreasing sequence of positive real numbers. Assume that there exist $0<K_{1}<K_{2}$ and $n \in \mathbf{N}$ such that $\forall k \leq n$,

$$
K_{1} x_{n}^{p+1} \leq\left(x_{n}-x_{n+1}\right) \leq K_{2} x_{n+1}^{p+1} .
$$

Then there exist $\tilde{K}_{1}$ and $\tilde{K}_{2}{ }^{7}$ such that for $\forall k \leq n$

$$
\tilde{K}_{1} \leq k^{\frac{1}{p}} x_{k} \leq \tilde{K}_{2} .
$$

Corollary 5.3. Let $\left(u_{n}\right)$ be a decreasing sequence of real numbers. Assume that there are $\alpha>0, \beta>0, p>0$ and $n \in \mathbf{N}$ such that $\forall k \leq n$

$$
\left(u_{k}-u_{k+1}\right) \leq \alpha+\beta e^{p u_{k+1}} \text {. }
$$

Then $\forall k \leq n$ we have

$$
\frac{\alpha^{\frac{1}{p}}}{\left(\alpha+\beta e^{p u_{0}}\right)^{\frac{1}{p}}} e^{-\alpha k} \leq e^{u_{k}-u_{0}} .
$$

Let us provide a short argument of how these corollaries can be deduced from the Lemma 5.1.

Proof. For Corollary 5.2 we use the lemma with the function $f: x \mapsto x^{-(p+1)}$ so that one may take $F: x \mapsto-\frac{1}{p} x^{-p}$. We deduce that we have

$$
K_{1} n \leq \frac{1}{p}\left(\frac{1}{x_{n}^{p}}-\frac{1}{x_{0}^{p}}\right) \leq K_{2} n .
$$

Elementary computations then lead to the desired inequalities.

For Corollary 5.3 we now consider the function $f: x \mapsto\left(1+\frac{\beta}{\alpha} e^{p x}\right)^{-1}$. One first checks that $F: x \mapsto x-\frac{1}{p} \log f(x)$ is an antiderivative of $f$. Our assumptions on $\left(u_{n}\right)$ may now be written as

$$
\left(u_{k}-u_{k+1}\right) f\left(u_{k}\right) \leq \alpha .
$$

Using Lemma 5.1 we deduce that $F\left(u_{0}\right)-F\left(u_{k}\right) \leq \alpha k$. This can be written in the form

$$
u_{0}-u_{k}+\frac{1}{p} \log \left(\frac{\alpha+\beta e^{p u_{k}}}{\alpha+\beta e^{p u_{0}}}\right) \leq \alpha k .
$$

Applying exponents to both sides of this last inequality, we deduce that

$$
\left(\frac{\alpha+\beta e^{p u_{k}}}{\alpha+\beta e^{p u_{0}}}\right)^{\frac{1}{p}} e^{-\alpha k} \leq e^{u_{k}-u_{0}} .
$$

From this we get our estimates.

5.1.2. Uniform estimates along the real axis. We now come back to our dynamical setting. Let $x_{0} \in I$ be a fixed element. Assume for convenience that $x_{0}<1$. Define for any $n \geq 0, f_{\varepsilon}\left(x_{n+1}(\varepsilon)\right)=x_{n}(\varepsilon)$, where $x_{0}(\varepsilon)=x_{0}$. For each $\varepsilon>0$ sufficiently small, we define $N_{\varepsilon}$ as $N_{\varepsilon}=\sup \left\{n \in \mathbf{N} \mid x_{n}^{p} \geq \varepsilon\right\}$, and for $\varepsilon=0$ as $N_{0}=+\infty$. Note that for any $\varepsilon>0$ small enough, the sequence $\left(x_{n}(\varepsilon)\right)$ is strictly

\footnotetext{
${ }^{7}$ One can take for instance $\tilde{K}_{2}=\left(p K_{1}\right)^{-\frac{1}{p}}$ and $\tilde{K}_{1}=\left(p K_{2}+\frac{1}{x_{0}^{p}}\right)^{-\frac{1}{p}}$.
} 
decreasing towards 0 . So that $N_{\varepsilon}$ is a well defined integer. Our main result in this paragraph is the following.

Proposition 5.4. There exists $K>1$ such that for all $\varepsilon>0$ small enough,

$$
\begin{aligned}
& K^{-1} \leq \varepsilon N_{\varepsilon} \leq K, \quad \varepsilon>0, \\
& K^{-1} \leq x_{n} n^{\frac{1}{p}} \leq K, \quad \forall n<N_{\varepsilon}, \\
& K^{-1} \leq x_{n} \varepsilon^{-\frac{1}{p}}(1+\varepsilon)^{n} \leq K, \quad \forall n \geq N_{\varepsilon} .
\end{aligned}
$$

This result may be interpreted in the following way: $N_{\varepsilon}$ is a "parabolic time". During that time, the fixed point 0 acts on the orbit of $x_{0},\left(x_{n}\right)$, as if it was a parabolic fixed point with $p$ petals. For $n$ greater than $N_{\varepsilon}$ the orbit of $x_{0}$ is close enough to 0 and realize that it is indeed an attracting fixed point for $f_{\varepsilon}^{-1}$.

In the following lemma we obtain estimates which are true for all $n \in \mathbf{N}$ and part of Proposition 5.4.

Lemma 5.5. There exists $K>1$ such that for all $\varepsilon>0$ small enough,

$$
K^{-1} \varepsilon^{\frac{1}{p}}(1+\varepsilon)^{-n} \leq x_{n} \leq K \frac{1}{n^{\frac{1}{p}}}, \quad \forall n \in \mathbf{N} .
$$

Proof. All our estimates will result from the following very definition of $\left(x_{n}\right)$,

$$
x_{n}=(1+\varepsilon) x_{n+1}+x_{n+1}^{p+1}\left(1+x_{n+1} g_{\varepsilon}\left(x_{n+1}\right)\right) .
$$

Assuming that $\varepsilon<1$, we easily deduce from this equality that for any $n$ we have

$$
1 \leq \frac{x_{n}}{x_{n+1}} \leq\left(2+2 x_{0}^{p}\right) \leq 4
$$

From (5.5) we deduce that

$$
\frac{x_{n}-x_{n+1}}{x_{n}^{p+1}}=\frac{\varepsilon x_{n+1}}{x_{n}^{p+1}}+\left(\frac{x_{n+1}}{x_{n}}\right)^{p+1}\left(1+x_{n+1} g_{\varepsilon}\left(x_{n+1}\right)\right),
$$

which, with (5.6) leads to

$$
K_{1}:=\frac{1}{4^{p+2}} \leq \frac{1}{2}\left(\frac{x_{n+1}}{x_{n}}\right)^{p+1} \leq \frac{x_{n}-x_{n+1}}{x_{n}^{p+1}} .
$$

Using now Corollary 5.2 we conclude that $\forall n, x_{n} n^{\frac{1}{p}} \leq\left(p \frac{1}{4^{p+2}}\right)^{\frac{-1}{p}} \leq 64$. This is precisely the right hand side of (5.4).

The left hand side of (5.4) is obtained when one notes that (5.5) also implies that

$$
\log x_{n}-\log x_{n+1}=\log \left(1+\varepsilon+x_{n+1}^{p}+x_{n+1}^{p+1} g_{\varepsilon}\left(x_{n+1}\right)\right) \leq \log (1+\varepsilon)+2 x_{n+1}^{p} .
$$

We may thus apply Corollary 5.3 with the sequence $u_{n}:=\log x_{n}, \alpha=\log (1+\varepsilon)$, and $\beta=2$, and deduce that

$$
\frac{\alpha^{\frac{1}{p}}}{\left(\alpha+\beta e^{p u_{0}}\right)^{\frac{1}{p}}} e^{-\alpha n} \leq e^{u_{n}-u_{0}} .
$$

Assuming that $\varepsilon$ is small enough so that $\frac{\varepsilon}{3} \leq \alpha=\log (1+\varepsilon) \leq e^{p u_{0}}$ we get

$$
\frac{1}{9} \varepsilon^{\frac{1}{p}}(1+\varepsilon)^{-n} \leq e^{u_{n}}=x_{n} .
$$

This ends the proof of Lemma 5.5. 
We are now in position to give a proof of Proposition 5.4, but first note that the right hand side of (5.2) and the left hand side of (5.3) are given by Lemma 5.5.

Proof. In order to get estimate (5.2), we check that the assumptions on $g_{\varepsilon}$, the definition of $N_{\varepsilon}$ and relation (5.7) leads for all $n<N_{\varepsilon}$ to

$$
\frac{x_{n}-x_{n+1}}{x_{n+1}^{p+1}} \leq \frac{5}{2}
$$

Corollary 5.2 then tells us that $\forall n<N_{\varepsilon}$ we have

$$
\tilde{K}_{1} \leq x_{n} n^{\frac{1}{p}}
$$

with, for instance, $\tilde{K}_{1}=\left(\frac{5 p}{2}+\frac{1}{x_{0}^{p}}\right)^{-\frac{1}{p}}$.

We are now in position to give estimates for $N_{\varepsilon}$. They easily come out from the following inequalities we have already proved:

$$
\frac{\tilde{K}_{1}}{K_{0}^{2 p}} \frac{1}{N_{\varepsilon}^{\frac{1}{p}}} \leq \frac{\tilde{K}_{1}}{K_{0}^{2 p}} \frac{1}{\left(N_{\varepsilon}-1\right)^{\frac{1}{p}}} \leq \frac{x_{N_{\varepsilon}-1}}{K_{0}^{2 p}} \leq \frac{x_{N_{\varepsilon}}}{K_{0}} \leq x_{N_{\varepsilon}+1} \leq \varepsilon^{\frac{1}{p}} \leq x_{N_{\varepsilon}} \leq \frac{2}{N_{\varepsilon}} .
$$

From there we deduce that

$$
\tilde{K}_{3} \leq \varepsilon^{\frac{1}{p}} N_{\varepsilon} \leq 2
$$

with $\tilde{K}_{3}=\frac{\tilde{K}_{1}}{K_{0}^{2 p}}$.

Now we only need to take care of (5.3). We start by noticing that for all $n$ we have $(1+\varepsilon) x_{n+1} \leq x_{n}$. For any $n \geq N_{\varepsilon}$ we thus have $(1+\varepsilon)^{n-N_{\varepsilon}} x_{n} \leq x_{N_{\varepsilon}}$. This leads to

$$
x_{n} \leq(1+\varepsilon)^{-n} x_{N_{\varepsilon}}(1+\varepsilon)^{N_{\varepsilon}} .
$$

By definition of $N_{\varepsilon}$ and relation (5.6) we have

$$
x_{N_{\varepsilon}} K_{0} x_{N_{\varepsilon}+1} \leq K_{0} \varepsilon^{\frac{1}{p}} .
$$

By relation (5.9) we also have

$$
(1+\varepsilon)^{N_{\varepsilon}} \leq\left(1+\frac{2^{p}}{N_{\varepsilon}}\right)^{N_{\varepsilon}} \leq e^{2^{p}} .
$$

From this and (5.10) we deduce that

$$
x_{n} \leq K_{0} e^{2^{p}} \varepsilon^{\frac{1}{p}}(1+\varepsilon)^{-n} .
$$

Taking $K=K_{0}^{2 p} e^{2^{p}}$ finishes the proof of the proposition.

Let now $\alpha(p):=\frac{p+1}{p}$. The following corollary is useful.

Corollary 5.6. There exists $K>1$ such that for all $\varepsilon>0$ small enough we have

(i) $K^{-1} \leq\left(x_{n}-x_{n+1}\right) n^{\alpha(p)} \leq K \quad \forall n \leq N_{\varepsilon}$,

(ii) $K^{-1} \leq\left(x_{n}-x_{n+1}\right) \varepsilon^{-\alpha(p)}(1+\varepsilon)^{(p+1) n} \leq K \quad \forall n>N_{\varepsilon}$.

Proof. From relation (5.5) we deduce that $\forall n \in \mathbf{N}$,

$$
x_{n}-x_{n+1}=\varepsilon x_{n+1}+x_{n+1}^{p+1}\left(1+x_{n+1} g_{\varepsilon}\left(x_{n+1}\right)\right),
$$

so that $\varepsilon x_{n+1} \leq x_{n}-x_{n+1}$, and Lemma 5.5 tells us that the left hand side inequality of (ii) is true. Moreover, for $n \leq N_{\varepsilon}$ we have $\varepsilon \leq x_{n+1}^{p}$, and (5.12) leads to $x_{n}-x_{n+1} \leq$ $3 x_{n+1}^{p+1}$. With Lemma 5.5, we get the right-hand side inequality of (i). 
Let $n<N_{\varepsilon}$. Then, from (5.2) and (5.6), we deduce that

$$
\frac{1}{2 K K_{0}^{p+1}} \frac{1}{n^{\alpha(p)}} \leq \frac{x_{n}^{p+1}}{K_{0}^{p+1}} \leq x_{n+1}^{p+1} \leq\left(x_{n}-x_{n+1}\right) .
$$

This is the left-hand side inequality of (i).

In order to finish fix $n \geq N_{\varepsilon}$. Then, by relation (5.5) and Proposition 5.4, we get that

$$
x_{n}-x_{n+1} \leq 3 x_{n+1}^{p+1} \leq 3 K^{p+1} \varepsilon^{\alpha(p)}(1+\varepsilon)^{-(p+1) n} .
$$

The proof of the corollary is now complete.

5.1.3. Extension to the complex plane. As already mentioned, this extension is done via Kobe's Distortion Theorem. It asserts that given two simply connected domains in $\mathbf{C}, V \subset V^{\prime}$, such that the boundary of $V$ is at a positive distance from the boundary of $V^{\prime}$, there exists a constant $K>0$, which depends only on the modulus of the annulus $V^{\prime} \backslash V$, and such that for any univalent function $f$ defined in $V^{\prime}$ we have for all $x, y \in V$ that

$$
\frac{1}{K} \leq \frac{\left|f^{\prime}(x)\right|}{\left|f^{\prime}(y)\right|} \leq K
$$

Proposition 5.7. Let $V$ be a domain such that $\bar{V} \subset \mathscr{U}_{\theta}$. Then there exists $K>0$ such that $\forall \varepsilon$ small enough, $\forall n \in \mathbf{N}$ and $\forall z \in V$ we have

(i) $\frac{1}{K} \leq n^{\alpha(p)}\left|\left(f_{\varepsilon}^{-n}\right)^{\prime}(z)\right| \leq K$ if $n<N_{\varepsilon}$,

(ii) $\frac{1}{K}(1+\varepsilon)^{-(p+1) n} \leq\left|\left(f_{\varepsilon}^{-n}\right)^{\prime}(z)\right| \leq K \varepsilon^{\alpha(p)}(1+\varepsilon)^{-(p+1) n}$ if $n \geq N_{\varepsilon}$.

Proof. Enlarging $V$ if necessary one may assume that there is $x_{0} \in V \cap \mathbf{R}^{+} \cap \mathscr{U}_{\theta}$ such that for all $\varepsilon$ small enough $x_{1}(\varepsilon):=f_{\varepsilon}^{-1}\left(x_{0}\right)$ is also in $V$. Kobe's distortion Theorem implies that for all $n$, all $\varepsilon$ and all $z \in V$ we have

$$
\frac{1}{K} \frac{\left(x_{n}(\varepsilon)-x_{n+1}(\varepsilon)\right)}{x_{0}-x_{1}(\varepsilon)} \leq\left|\left(f_{\varepsilon}^{-n}\right)^{\prime}(z)\right| \leq K \frac{\left(x_{n}(\varepsilon)-x_{n+1}(\varepsilon)\right)}{x_{0}-x_{1}(\varepsilon)} .
$$

Applying Corollary 5.6, and noticing that $x_{0}-x_{1}(\varepsilon)>a>0$ with some real $a$ independent of $\varepsilon$, leads to the desired inequalities.

The following result gives uniform estimates on how closely the orbits are tangent to the real axis.

Corollary 5.8. There exists $K>0$ such that $\forall \varepsilon$ small enough, $\forall n \in \mathbf{N}$ and $\forall z_{0} \in V$, we have

In particular, the series $\sum_{n=0}^{\infty} \operatorname{Im}\left(f_{\varepsilon}^{-n}\left(z_{0}\right)\right)$ converges.

$$
\left|\operatorname{Im}\left(f_{\varepsilon}^{-n}\left(z_{0}\right)\right)\right| \leq K \frac{1}{n^{\alpha(p)}} .
$$

Proof. Note that $\left|\operatorname{Im}\left(z_{n}\right)\right|=\left|\operatorname{Im}\left(z_{n}-x_{n}\right)\right| \leq\left|z_{n}-x_{n}\right|$. Kobe's distortion theorem leads to $\left|z_{n}-x_{n}\right| \leq K \frac{1}{\left|\left(f_{\varepsilon}^{n}\right)^{\prime}\left(z_{n}\right)\right|}$ and Proposition 5.7 gives the result.

5.2. Estimates of some partial sums. In this appendix we single out the behaviour of the partial sums we need to evaluate at several steps in the proof of our main result. We are thus in this paragraph dealing with a sequence of real numbers defined by $a_{n}=1 / n$ for $n \leq N_{\varepsilon}$ and $a_{n}=\varepsilon(1+\varepsilon)^{-n}$ for $n>N_{\varepsilon}$, where $N_{\varepsilon}$ is comparable with $1 / \varepsilon$. We are indeed interested in the sequences $\left(a_{n}(\alpha)\right)_{n \in \mathbf{N}}$, with $\alpha \in \mathbf{R}$ and $a_{n}(\alpha)=a_{n}^{\alpha}$, and partial sums $S_{k, n}(\alpha)=\sum_{j=k}^{n} a_{j}(\alpha)$. 
The first lemma, whose proof is straightforward and left to the reader asserts, the following.

Lemma 5.9. For any $k<n$ in $\mathbf{N}$ we have

$$
\begin{aligned}
& S_{k, n}(\alpha) \sim \begin{cases}\frac{1}{1-\alpha}\left(n^{1-\alpha}-k^{1-\alpha}\right) & \text { if } n \leq N_{\varepsilon} \text { and } \alpha \neq 1, \\
\log \frac{n}{k} & \text { if } n \leq N_{\varepsilon} \text { and } \alpha=1,\end{cases} \\
& S_{k, n}(\alpha) \sim \frac{1}{\alpha \varepsilon}\left(a_{k}(\alpha)-a_{n}(\alpha)\right) \\
& \text { if } k>N_{\varepsilon} \text { and } \alpha \neq 0 .
\end{aligned}
$$

As its consequence, we get the following.

Corollary 5.10. If $\alpha>0$ then

(i) $S_{n,+\infty}(\alpha) \sim \frac{a_{n}(\alpha)}{\varepsilon}$ if $n>N_{\varepsilon}$,

(ii) $S_{n,+\infty}(\alpha) \sim a_{n}(\alpha-1)$ if $n \leq N_{\varepsilon}$ and $\alpha>1$,

(iii) $S_{n,+\infty}(\alpha) \sim \log \frac{N_{\varepsilon}}{n}+K$ if $n \leq N_{\varepsilon}, \alpha=1$, for some $K>0$,

(iv) $S_{n,+\infty}(\alpha) \sim N_{\varepsilon}^{1-\alpha}$ if $n \leq N_{\varepsilon}$ and $\alpha<1$.

Proof. Since $\alpha>0$, we see that the sequence $(1+\varepsilon)^{-\alpha n}$ converges to 0 , and Lemma 5.9 implies that (i) is true. Note that we have

$$
\max \left(S_{n, N_{\varepsilon}}(\alpha), S_{N_{\varepsilon},+\infty}(\alpha)\right) \leq S_{n,+\infty} \leq 2 \max \left(S_{n, N_{\varepsilon}}(\alpha), S_{N_{\varepsilon},+\infty}(\alpha)\right) .
$$

Using (i) that we have just proved, the fact that we have $a_{N_{\varepsilon}} \sim a_{N_{\varepsilon}+1}$, and the fact that $N_{\varepsilon} \sim \varepsilon^{-1}$, we conclude that

$$
S_{N_{\varepsilon},+\infty} \sim \frac{a_{N_{\varepsilon}}}{\varepsilon} \sim \varepsilon^{\alpha-1} \sim N_{\varepsilon}^{1-\alpha} .
$$

Let us now estimate $S_{n, N_{\varepsilon}}$ by considering three cases. We start with the case when $\alpha=1$. Indeed, Lemma 5.9 implies that $S_{n, N_{\varepsilon}} \sim \log \left(\frac{N_{\varepsilon}}{n}\right)$. This gives us (iii).

Assume now that $\alpha>1$. Then $S_{N_{\varepsilon},+\infty} \sim N_{\varepsilon}^{1-\alpha} \leq n^{1-\alpha}=a_{n}(\alpha-1)$. Moreover, in virtue of Lemma 5.9, we have $S_{n, N_{\varepsilon}} \sim n^{1-\alpha}-N_{\varepsilon}^{1-\alpha}$. Thus

$$
S_{n, N_{\varepsilon}} \sim a_{n}(\alpha-1)\left(1-\left(\frac{n}{N_{\varepsilon}}\right)^{\alpha-1}\right) .
$$

In particular $S_{n, N_{\varepsilon}} \lesssim a_{n}(\alpha-1)$. So, we can conclude that $S_{n,+\infty}(\alpha) \lesssim a_{n}(\alpha-1)$. If $\frac{n}{N_{\varepsilon}} \leq \frac{1}{2}$, we have $\left(1-\frac{n}{N_{\varepsilon}}\right)^{\alpha-1} \geq\left(1-\frac{1}{2}\right)^{\alpha-1}$. And we also have $S_{n,+\infty}(\alpha) \gtrsim a_{n}(\alpha-1)$; so, we are done. On the other hand, if $\frac{n}{N_{\varepsilon}} \geq \frac{1}{2}$, then

$$
S_{n,+\infty}(\alpha) \geq S_{N_{\varepsilon},+\infty}(\alpha) \sim N_{\varepsilon}^{1-\alpha} \sim n^{1-\alpha}=a_{n}(\alpha-1) .
$$

This ends the proof of (ii).

Assume finally that $0<\alpha<1$. Then Lemma 5.9 tells us that

$$
S_{n, N_{\varepsilon}}(\alpha) \sim\left(N_{\varepsilon}^{1-\alpha}-n^{1-\alpha}\right) \leq N_{\varepsilon}^{1-\alpha} \sim S_{N_{\varepsilon}, \infty} .
$$

We thus conclude that $\max \left(S_{n, N_{\varepsilon}}(\alpha), S_{N_{\varepsilon},+\infty}(\alpha)\right) \sim S_{N_{\varepsilon},+\infty}$. This proves (iv) and ends the proof of the corollary.

We can also prove the following result with the same kind of arguments. So we omit them. 
Corollary 5.11. Let $N$ be a fixed integer such that $2 N<N_{\varepsilon} \sim \frac{1}{\varepsilon}$. Then we have the following estimates of $S_{N, n}(\alpha)$ for $N \leq n$ :

$$
S_{N, n}(\alpha) \sim\left\{\begin{array}{cc}
a_{N}(\alpha-1)-a_{n}(\alpha-1) & \text { for } 1<\alpha \\
\log \frac{n}{N} & \text { for } \alpha=1 \\
a_{n}(\alpha-1)-a_{N}(\alpha-1) & \text { for } \alpha<1
\end{array}\right\} \text { for } n \leq N_{\varepsilon},
$$

\section{References}

[Bo,Zi] Bodart, O., and M. Zinsmeister: Quelques résultats sur la dimension de Hausdorff des ensembles de Julia des polynômes quadratiques. - Fund. Math. 151:2, 1996, 121-137.

[Bu,Le] Buff, X., and L. TAn: Dynamical convergence and polynomial vector fields. - J. Differential Geom. 77:1, 2007, 1-41.

[Do,Se,Zi] Doundy, A., P. Sentenac, and M. Zinsmeister: Implosion parabolique et dimension de Hausdorff. - C. R. Acad. Sci. Paris Sér. I Math. 325:7, 1997, 765-772.

[Ha] Havard, G. Applications du formalisme thermodynamique à l'étude de certains ensembles de Julia. - Thèse de l'université d'Orléans, 1999.

$\left[\mathrm{Ha}_{2} \mathrm{Zi}^{1}\right]$ HaVARD, G., and M. Zinsmeister: Thermodynamic formalism and variations of the Hausdorff dimension of quadratic Julia sets. - Comm. Math. Phys. 210:1, 2000, 225-247.

[Ha,Se,Zi] Havard, G., P. Sentenac, and M. Zinsmeister: Le chou-fleur a une dimension de Hausdorff inft'erieure 'a 1,295. - Preprint, 2000.

[He,Ur] Heinemann, S., and M. URBAŃski: Hausdorff dimension estimates for infinite conformal iterated function systems. - Nonlinearity 15, 2002, 727-734.

$\left[\mathrm{Ja}^{1}\right] \quad$ JakszTAs, L.; Derivative of the Hausdorff dimension of the Julia set. - Preprint, 2008.

$\left[\mathrm{Ja}^{2}\right] \quad$ JakszTas, L.: Personal communication.

[Je,Po] Jenkinson, O., and M. Pollicott: Computing the dimension of dynamically defined sets: E2 and bounded continued fractions. - Ergodid Theory Dynam. Systems 21, 2001, 1429-1445.

[Ma] Martens, M.: The existence of sigma-finite invariant measures, applications to real one-dimensional dynamics. - arXiv:math/9201300.

[Ma,Ur $\left.{ }^{1}\right]$ MAYER, V., and M. URBAŃSKi: Geometric thermodynamical formalism and real analyticity for meromorphic functions of finite order. - Ergodic Theory Dynam. Systems 28, 2008, 915-946.

[Ma, Ur $\left.{ }^{2}\right]$ MAYER, V. and M. URBAŃski: Thermodynamical formalism and multifractal analysis for meromorphic functions of finite order. - Mem. Amer. Math. Soc. 203:954, 2010.

$\left[\mathrm{McMu}^{1}\right]$ MCMullen, C.: Area and Hausdorff dimension of Julia sets of entire functions. - Trans. Amer. Math. Soc. 300, 1987, 329-342.

$\left[\mathrm{McMu}^{2}\right]$ MCMullen, C.: Hausdorff dimension and conformal dynamics III: Computation of dimension. - Amer. J. Math. 120, 1998, 691-721.

$\left[\mathrm{McMu}^{3}\right]$ MCMullen, C.: Hausdorff dimension and conformal dynamics. I. Strong convergence of Kleinian groups. - J. Differential Geom. 51:3, 1999, 471-515.

$\left[\mathrm{McMu}^{4}\right]$ MCMullen, C.: Hausdorff dimension and conformal dynamics. II. Geometrically finite rational maps. - Comment. Math. Helv. 75:4, 2000, 535-593. 
[Re,Si] ReED, M., and B. Simon: Methods of modern mathematical physics, functional analysis I and analysis of operators IV. - Academic Press, NY, 1978.

[Ri] Rivera-Letelier, J.: On the continuity of Hausdorff dimension of Julia sets and similarity between the Mandelbrot set and Julia sets. - Fund. Math. 170:3, 2001, 287-317.

[Ru $\quad$ Ruelle, D.: Repellers for real analytic maps. - Ergodic Theory Dynam. Systems 2:1, 1982, 99-107.

[Su] Sullivan, D.: Conformal dynamical systems. - In: Geometric dynamics (Rio de Janeiro, 1981), Lecture Notes in Math. 1007, 725-752.

[Ur] Urbański, M.: Thermodynamic formalism and multifractal analysis of finer Julia sets of exponential family. - Preprint.

[Ur,Zd $\left.{ }^{1}\right]$ Urbański, M., and A. Zdunik: Real analyticity of Hausdorff dimension of finer Julia sets of exponential family. - Ergodig Theory Dynam. Systems 24, 2004, 279-315.

[Ur,Zd $\left.{ }^{2}\right]$ Urbański, M., and A. Zdunik: The parabolic map $\frac{1}{e} e^{z}$. - Indag. Math. (N.S.) 15, 2004, 419-433.

[Ur,Zi $\left.{ }^{1}\right]$ Urbański, M., and M. Zinsmeister: Continuity of Hausdorff dimension of JuliaLavaurs sets as a function of the phase. - Conform. Geom. Dyn. 5, 2001, 140-152.

[Ur,Zi $\left.{ }^{2}\right]$ Urbański, M., and M. Zinsmeister: Parabolic implosion and Julia-Lavaurs sets in the exponential family. - Monatsh. Math. 149:2, 2006, 129-140.

Received 27 October 2008 